Homology, Homotopy and Applications, vol.4(2), 2002, pp.227-258

\title{
KOSZUL HOMOLOGY AND LIE ALGEBRAS WITH APPLICATION TO GENERIC FORMS AND POINTS
}

\author{
R. FRÖBERG AND C. LÖFWAL \\ (communicated by Larry Lambe)
}

\begin{abstract}
We study the Koszul dual for general superalgebras, and apply it to the Koszul homology of a graded algebra. We show that a part of the Koszul homology algebra is related to the homotopy Lie algebra by means of Koszul duality. This is used to study the "Minimal Resolution Conjecture" and the "Ideal Generating Conjecture" for sets of generic points in projective space, and for quotients of the polynomial ring (or exterior algebra) modulo generic quadratic forms.
\end{abstract}

\section{To Jan-Erik Roos on his sixty-fifth birthday}

\section{Introduction}

Consider $s$ generic points in projective space $P_{k}^{n}$. The Hilbert series of the corresponding coordinate ring $A=S / I=k\left[x_{0}, \ldots, x_{n}\right] / I$ is known to be $\sum_{i \geqslant 0} \min \left(\left(\begin{array}{c}n+i \\ i\end{array}\right), s\right) z^{i}$ [Ge-Or 81]. The Koszul homology of $A, H=\operatorname{Tor}^{S}(A, k)$, is known to satisfy $H_{i, j}=0$ if $j \neq d+i-1, d+i$ for a certain $d$ (depending on $n$ and $s$ ), but there are a lot of unanswered questions. The Minimal Resolution Conjecture (MRC) states that for all $i$ and all $j, H_{i, j}=0$ or $H_{i+1, j}=0$, and the special case that this is true for $i=1$ is called the Ideal Generating Conjecture (IGC). The MRC has recently been disproved [Ei-Po 99], while IGC is still open. In this paper we will introduce a new approach to study these problems, namely through the "homotopy Lie algebra", $\mathfrak{g}_{A}$, of $A$.

The MRC may be reformulated in terms of $\mathfrak{g}_{A}$. In fact, there are certain numbers $m$ and $t$ (depending on $n$ and $s$ ) such that if $d>2$ then MRC holds if and only if $\operatorname{dim}_{k} \mathfrak{g}_{A}^{m+1, m+d-1}=t$ and $\mathfrak{g}_{A}^{m+2, m+d}=0$. When $d=2$ the situation is a bit more complicated. Let $\eta_{A}$ be the "diagonal" Lie algebra of $\mathfrak{g}_{A}$; i.e., $\eta_{A}^{i}=\mathfrak{g}_{A}^{i, i}$ and let $L=\oplus_{i \geqslant 2} \eta_{A}^{i}$ and finally $Q^{i}=(L /[L, L])^{i}$. Then if $d=2$, MRC holds if and only if $\operatorname{dim}_{k} Q^{m+1}=t$ and $Q^{m+2}=0$.

Even if the case $d>2$ is easier, we do not know any application of it. However in the case when $d=2$, the Lie algebra $\eta_{A}$ is explicitly given as the free Lie algebra on $n+1$ odd variables modulo the ideal generated by the squares of $s$ generic linear forms. We have used a Mathematica program called "liedim" and its C-version

Received June 2, 2000, revised September 12, 2000; published on July 12, 2002. 2000 Mathematics Subject Classification: 13D07, 14M99, 16E45.

Key words and phrases: Koszul homology, Lie algebra, generic points, generic forms. (c) 2002, R. Fröberg and C. Löfwal. Permission to copy for private use granted. 
"cbas" to compute the space $Q$ above for some values of $n$ and $s$. In this way we have been able to prove MRC and IGC in some new cases.

We have also studied ideals generated by generic quadratic forms in polynomial rings and exterior algebras and ideals generated by generic quadratic Lie elements in free Lie algebras.

The paper is organized as follows. In Section 2 the Koszul dual is defined for general superalgebras, in particular for graded commutative algebras. This will be applied to the Koszul homology algebra, which has generators of both even and odd degrees. In Section 3 the homotopy Lie algebra is introduced for graded commutative algebras through minimal models and in Section 4 a theorem is proved which relates parts of the Koszul homology algebra and the homotopy Lie algebra by means of Koszul duality. In fact, since we are dealing with superalgebras, our theorem may also be applied to quotients of exterior algebras (or to quotients of tensor products of exterior algebras and polynomial algebras). However, in the exterior algebra case, $\operatorname{Tor}^{S}(A, k)$ is in general infinite-dimensional and the corresponding Lie algebra is an "ordinary" Lie algebra (non-super).

In Section 5 we introduce the MRC and IGC conjectures and apply the theorem in Section 4 in order to reformulate the conjectures in terms of the Lie algebra $L$ associated to $A$. We also prove a "monotonicity" property, which implies that if counterexamples to MRC exists for a given $n$, then there has to be counterexamples for special values of $s$. For IGC this holds for at most two values of $s$ for each $n$. In Section 6 we present some theorems about MRC and IGC obtained by computations and by the theory from the previous section. The computations are presented in Section 11.

In Section 7 we study rings of the form $k\left[x_{1}, \ldots, x_{n}\right] /\left(f_{1}, \ldots, f_{r}\right)$, where $f_{1}, \ldots, f_{r}$ are generic quadratic forms. We prove that the ring is Koszul if and only if $r \leqslant n$

or $r \geqslant\left(\begin{array}{c}n+1 \\ 2\end{array}\right)-n^{2} / 4$. The corresponding result for Lie algebras is also obtained. We study the series of Lie algebras obtained from generic quadratic algebras or defined by generic quadratic Lie relations. We make some conjectures and prove them in some special cases. In Section 8 we state a conjecture about the Lie algebra for the coordinate ring of a set of generic points, and we prove it in some cases. In Section 9 we study the Poincaré series for these coordinate rings. In Section 10 finally, we study the quotient of exterior algebras with generic quadratic forms and their corresponding (ordinary) Lie algebras. In particular we study the exterior algebra in 5 variables modulo 2 or 3 generic quadratic forms. The Hilbert series turned out to be not the ones which were expected.

\section{The Koszul dual}

We will consider $\mathbb{Z}_{2} \times \mathbb{N}$-graded (or $\mathbb{Z}_{2} \times \mathbb{N} \times \mathbb{N}$-graded) associative algebras or Lie algebras over a field $k$ of characteristic different from two. The $\mathbb{Z}_{2}$-grading (also called the "parity") of an element $x$ is denoted $|x|$ and we ususally talk about "even" and "odd" elements corresponding to whether $|x|=0$ or $|x|=1$. This grading makes our algebras "superalgebras", and it is this grading that determines the sign when two elements in a formula are interchanged; e.g., the graded commutator is 
defined as $[a, b]=a b-(-1)^{|a||b|} b a$ and the graded Jacobi identity is $[[a, b], c]=$ $[a,[b, c]]+(-1)^{|b||c|}[[a, c], b]$. The additional $\mathbb{N}$-gradings are called "weights" and they have no effect on signs in formulas.

The Koszul dual $A^{!}$of a $\mathbb{Z}_{2} \times \mathbb{N}$-graded connected $k$-algebra $A=k \oplus A_{1} \oplus A_{2} \oplus \cdots$ is again a $\mathbb{Z}_{2} \times \mathbb{N}$-graded connected $k$-algebra and may be defined as the subalgebra of $\operatorname{Ext}_{A}(k, k)$ generated by the elements of homological degree and weight equal to one. The algebra $A$ is called a Koszul algebra if $A^{!}=\operatorname{Ext}_{A}(k, k)$. The Koszul dual may be computed using the cobar construction $\left(\mathrm{T}\left(\left(s A_{+}\right)^{*}\right), d\right)$, cf [Ad 60]. Here $A_{+}$is the set of elements of positive weight, which is considered to be concentrated in homological degree zero, $\mathrm{T}$ stands for "tensor algebra" and $s$ is the suspension functor, which changes parity and raises the homological degree by one, while the weight is left unchanged by $s$. The differential $d$ is the derivation which extends the map

$$
\left(s A_{+}\right)^{*} \stackrel{s}{\rightarrow} A_{+}^{*} \stackrel{m^{*}}{\rightarrow}\left(A_{+} \otimes A_{+}\right)^{*} \stackrel{\left(\phi_{A_{+}}\right)^{-1}}{\longrightarrow}\left(s A_{+}\right)^{*} \otimes\left(s A_{+}\right)^{*},
$$

where $m: A_{+} \otimes A_{+} \rightarrow A_{+}$is multiplication and $\phi_{A_{+}}$is a natural isomorphism $\phi_{V}$ applied to $V=A_{+}$. It is defined as follows. Let $V$ be a $\mathbb{Z}_{2} \times \mathbb{N}$-graded vector space, then $\phi_{V}:(s V)^{*} \otimes(s V)^{*} \rightarrow(V \otimes V)^{*}$ is defined by $\phi(f \otimes g)=(f \otimes g) \circ(s \otimes s)$ for $f, g \in(s V)^{*}$. Following the strict sign rule, this means that the following holds for $a, b \in V$.

$$
\begin{aligned}
& (\phi(f \otimes g))(a \otimes b)=(-1)^{|a|+|g||s a|} f(s a) g(s b)= \\
& \quad(-1)^{|a|+|f||g|} f(s a) g(s b)=-(-1)^{|s a||b|} f(s a) g(s b) .
\end{aligned}
$$

It is easy to prove ( $\mathrm{cf}[\mathbf{P r} \mathbf{7 0}],[\mathbf{L o ̈ ~} \mathbf{8 6}])$ that

$$
A^{!}=\mathrm{T}\left(\left(s A_{1}\right)^{*}\right) /\left\langle d\left(\left(s A_{2}\right)^{*}\right)\right\rangle \text {. }
$$

Here $A$ ! only depends on the "1-2-algebra of $A$ ",

$$
\mathrm{T}\left(A_{1}\right) /\left\langle\operatorname{ker}\left(A_{1} \otimes A_{1} \rightarrow A_{2}\right)\right\rangle,
$$

which in fact is equal to $\left(A^{!}\right)$!

This gives the following description of the Koszul dual. Let $V$ be a $\mathbb{Z}_{2}$-graded finite-dimensional (or locally finite-dimensional $\mathbb{Z}_{2} \times \mathbb{N}$-graded) vector space and $R$ a homogeneous subspace of $V \otimes V$. Then

$$
(\mathrm{T}(V) /\langle R\rangle)^{!}=\mathrm{T}\left((s V)^{*}\right) /\left\langle\phi_{V}^{-1} R^{\perp}\right\rangle,
$$

where $s$ is the parity switcher, $R^{\perp}=\left\{f \in(V \otimes V)^{*} ; f(R)=0\right\}$ and $\phi_{V}:(s V)^{*} \otimes$ $(s V)^{*} \rightarrow(V \otimes V)^{*}$ is the isomorphism defined above. In coordinates this means the following (we have changed the formulas by a global -1 ). If $\{x, y, \ldots\}$ is a basis for $V$ and $\{X, Y, \ldots\}$ is a basis for $(s V)^{*}$ dual to $\{s x, s y, \ldots\}$, then an element $X \otimes Y$ is considered to operate on a basis element $a \otimes b$ by the rule $(X \otimes Y)(a \otimes b)=0$ if $a \neq x$ or $b \neq y,(X \otimes X)(x \otimes x)=1$ and

$$
(X \otimes Y)(x \otimes y)=\left\{\begin{aligned}
-1 & \text { if } x \text { is even and } y \text { is odd } \\
1 & \text { otherwise. }
\end{aligned}\right.
$$


Observe that this somewhat strange rule follows from the fact that the suspension $s$ has to pass $x$ before $Y$ passes $s x$.

Example 2.1. Let $|x|=0,|y|=1$. Then

$$
\left(k\langle x, y\rangle /\left\langle x y-y x, y^{2}\right\rangle\right)^{!}=k\langle X, Y\rangle /\left\langle X Y-Y X, X^{2}\right\rangle,
$$

where $|X|=1,|Y|=0$.

This example is in accordance with the known fact that the Ext-algebra of a free graded commutative algebra is free graded commutative with a parity switch (cf. [Ma 88] 3.10). See Proposition 2.2 below.

For a $\mathbb{Z}_{2}$-graded vector space $V$, let $[V, V]_{\mathcal{F}}$ denote the subspace of $V \otimes V$ generated by the graded commutators. The subscript $\mathcal{F}$ refers to the fact that $[V, V]_{\mathcal{F}}$ is contained in $\mathcal{F}(V)$, the free Lie algebra on $V$, considered as the Lie subalgebra generated by $V$ in the free associative algebra $\mathrm{T}(V)$. Then $\mathrm{U}(\mathcal{F}(V))=\mathrm{T}(V)$, where $\mathrm{U}$ stands for "universal enveloping algebra". The free graded commutative algebra on $V$, denoted $\bigwedge(V)$, is defined as $\mathrm{T}(V) /\left\langle[V, V]_{\mathcal{F}}\right\rangle$. Now $V \wedge V=(V \otimes V) /[V, V]_{\mathcal{F}}$ and hence $(V \wedge V)^{*}$ may (and will) be identified with $\left([V, V]_{\mathcal{F}}\right)^{\perp} \subset(V \otimes V)^{*}$.

Proposition 2.2. Let $V$ be a $\mathbb{Z}_{2} \times \mathbb{N}$-graded locally finite-dimensional vector space and let $s$ be the parity switcher. Let $R$ be a subspace of $V \otimes V$ which contains $[V, V]_{\mathcal{F}}$ and let $\bar{R}=R /[V, V]_{\mathcal{F}}$. Consider the map $\phi_{V}=\phi:(s V)^{*} \otimes(s V)^{*} \rightarrow(V \otimes V)^{*}$ defined (as above) by $\phi(f \otimes g)(a \otimes b)=(-1)^{|a|+|g||s a|} f(s a) g(s b)$. Then

$$
\begin{aligned}
& (i) \phi\left[(s V)^{*},(s V)^{*}\right]_{\mathcal{F}}=\left([V, V]_{\mathcal{F}}\right)^{\perp} \\
& (i i)(\bigwedge(V) /\langle\bar{R}\rangle)^{!} \cong \mathrm{U}\left(\mathcal{F}\left((s V)^{*}\right) /\left\langle\phi^{-1} R^{\perp}\right\rangle\right) \\
& (i i i)(\bigwedge(V))^{!} \cong \bigwedge\left((s V)^{*}\right) \\
& (i v) \bar{R}^{*} \cong\left[(s V)^{*},(s V)^{*}\right]_{\mathcal{F}} / \phi^{-1} R^{\perp} .
\end{aligned}
$$

Proof. (i). Let $f, g \in(s V)^{*}$ and $a, b \in V$. Then

$$
\begin{aligned}
& \phi(f \otimes g)\left(a \otimes b-(-1)^{|a||b|} b \otimes a\right)= \\
& (-1)^{|a||b|+|b|+1} f(s a) g(s b)-(-1)^{|a|+1} f(s b) g(s a) \quad \text { and } \\
& \phi(g \otimes f)\left(a \otimes b-(-1)^{|a||b|} b \otimes a\right)= \\
& (-1)^{|f||g|+|a|} g(s a) f(s b)-(-1)^{|f||g|+|a||b|+|b|} g(s b) f(s a) .
\end{aligned}
$$

Hence $\phi(f \otimes g)-(-1)^{|f||g|} \phi(g \otimes f)$ is zero on $a \otimes b-(-1)^{|a||b|} b \otimes a$ and hence $\phi\left[(s V)^{*},(s V)^{*}\right]_{\mathcal{F}} \subset\left([V, V]_{\mathcal{F}}\right)^{\perp}$. The other inclusion follows by dimension reasons.

(ii). This follows directly from $(i)$ and the description of the Koszul dual above, since $\bigwedge(V) /\langle\bar{R}\rangle \cong \mathrm{T}(V) /\langle R\rangle$ and

$$
\mathrm{T}\left((s V)^{*}\right) /\left\langle\phi^{-1} R^{\perp}\right\rangle \cong \mathrm{U}\left(\mathcal{F}\left((s V)^{*}\right) /\left\langle\phi^{-1} R^{\perp}\right\rangle\right),
$$

which is true since, $\phi^{-1} R^{\perp} \subset \phi^{-1}\left([V, V]_{\mathcal{F}}\right)^{\perp}=\left[(s V)^{*},(s V)^{*}\right]_{\mathcal{F}}$.

(iii). This follows from $(i)$ and $(i i)$ with $R=[V, V]_{\mathcal{F}}$. 
(iv). Using (i) we get

$$
\begin{aligned}
\bar{R}^{*}= & \left(R /[V, V]_{\mathcal{F}}\right)^{*} \cong\left([V, V]_{\mathcal{F}}\right)^{\perp} / R^{\perp} \cong \\
& \phi^{-1}\left([V, V]_{\mathcal{F}}\right)^{\perp} / \phi^{-1} R^{\perp}=\left[(s V)^{*},(s V)^{*}\right]_{\mathcal{F}} / \phi^{-1} R^{\perp} .
\end{aligned}
$$

In general, for an augmented $k$-algebra $A$, the subalgebra of $\operatorname{Ext}_{A}(k, k)$ generated by $\operatorname{Ext}_{A}^{1}(k, k)$ has relations of degree greater than two. However the relations of degree two may be described explicitly.

Proposition 2.3. Let $A$ be an augmented $k$-algebra with augmentation ideal $I$ and let $\operatorname{gr}(A)=k \oplus I / I^{2} \oplus I^{2} / I^{3} \oplus \cdots$ be the graded associated $k$-algebra. Then the 1-2-algebra of $\operatorname{Ext}_{A}(k, k)$ is isomorphic to the Koszul dual of $\operatorname{gr}(A)$; i.e.,

$$
\left(\operatorname{Ext}_{A}(k, k)^{!}\right)^{!} \cong(\operatorname{gr}(A))^{!} .
$$

Proof. All we need is to consider the beginning of the cobar construction, $(s I)^{*} \stackrel{d}{\rightarrow}$ $(s I)^{*} \otimes(s I)^{*}$, where $d$ is defined as above. We have $\operatorname{Ext}_{A}^{1}(k, k)=\left(s I^{2}\right)^{\perp}$. Hence

$$
\operatorname{ker}\left(\operatorname{Ext}_{A}^{1}(k, k) \otimes \operatorname{Ext}_{A}^{1}(k, k) \rightarrow \operatorname{Ext}_{A}^{2}(k, k)\right)=\operatorname{im}(d) \cap\left(\left(s I^{2}\right)^{\perp} \otimes\left(s I^{2}\right)^{\perp}\right) .
$$

With $\phi$ and $m$ as above we have $\phi(\operatorname{im}(d))=(\operatorname{ker}(m))^{\perp}$ and

$$
\phi\left(\left(s I^{2}\right)^{\perp} \otimes\left(s I^{2}\right)^{\perp}\right)=\left(I^{2} \otimes I+I \otimes I^{2}\right)^{\perp} .
$$

Hence

$$
\phi \operatorname{ker}\left(\operatorname{Ext}_{A}^{1}(k, k) \otimes \operatorname{Ext}_{A}^{1}(k, k) \rightarrow \operatorname{Ext}_{A}^{2}(k, k)\right)=\left(I^{2} \otimes I+I \otimes I^{2}+\operatorname{ker}(m)\right)^{\perp}
$$

which equals $\left(m^{-1}\left(I^{3}\right)\right)^{\perp}$, since $m\left(I^{2} \otimes I\right)=I^{3}$. Thus the 1-2-algebra of $\operatorname{Ext}_{A}(k, k)$ is isomorphic to $\mathrm{T}\left(\left(s I^{2}\right)^{\perp}\right) /\left\langle\phi^{-1}\left(m^{-1}\left(I^{3}\right)\right)^{\perp}\right\rangle$.

On the other hand

$$
(\operatorname{gr}(A))^{!}=\mathrm{T}\left(\left(s I / I^{2}\right)^{*}\right) /\left\langle\phi_{I / I^{2}}^{-1}\left(\operatorname{ker}\left(I / I^{2} \otimes I / I^{2} \rightarrow I^{2} / I^{3}\right)\right)^{\perp}\right\rangle
$$

Looking at $\left(I / I^{2} \otimes I / I^{2}\right)^{*}$ as a subspace of $(I \otimes I)^{*}$, the space $\left(\operatorname{ker}\left(I / I^{2} \otimes I / I^{2} \rightarrow I^{2} / I^{3}\right)\right)^{\perp}$ will be identified with $\left(m^{-1}\left(I^{3}\right)\right)^{\perp}$. Hence

$$
(\operatorname{gr}(A))^{!} \cong \mathrm{T}\left(\left(s I^{2}\right)^{\perp}\right) /\left\langle\phi_{I}^{-1}\left(m^{-1}\left(I^{3}\right)\right)^{\perp}\right\rangle .
$$

We end this section with a proposition which, combined with Proposition 2.3, will be used in Section 4 .

Proposition 2.4. Let $L$ be a $\mathbb{Z}_{2} \times \mathbb{N}^{+}$-graded Lie algebra, locally finite-dimensional over $k$. Let $\operatorname{gr}(L)$ denote the graded associated Lie algebra with respect to the filtration $L \supset[L, L] \supset[L,[L, L] \supset \cdots$ and let $\operatorname{gr}(\mathrm{U}(L))$ denote the graded associated algebra with respect to the filtration obtained from powers of the augmentation ideal. Then $\operatorname{gr}(\mathrm{U}(L))$ and $\mathrm{U}(\operatorname{gr}(L))$ are naturally isomorphic as graded algebras. 
Proof. There is a natural map of Lie algebras from $\operatorname{gr}(L)$ to the Lie algebra associated to the algebra $\operatorname{gr}(\mathrm{U}(L))$ and hence there is induced a natural map of graded algebras $\mathrm{U}(\operatorname{gr}(L)) \rightarrow \operatorname{gr}(\mathrm{U}(L))$. This map is surjective, since both algebras are generated by $L /[L, L]$ (depending on the assumption that $L$ has a positive grading). Since, by assumption, both algebras are locally finite-dimensional, it follows that the map is an isomorphism, since the algebras have the same Hilbert series.

\section{The homotopy Lie algebra}

Let $V$ be a $\mathbb{Z}_{2} \times \mathbb{N}^{+}$-graded vector space over $k$ with basis $\left\{x_{1}, \ldots, x_{n}\right\}$. We will also denote the free graded commutative algebra on $V, \bigwedge(V)$, by $k\left[x_{1}, \ldots, x_{n}\right]$. It is the free graded associative algebra $k\left\langle x_{1}, \ldots, x_{n}\right\rangle$ modulo the graded commutators $x_{i} x_{j}-(-1)^{\left|x_{i}\right|\left|x_{j}\right|} x_{j} x_{i}$. Sometimes $V$ will be infinite-dimensional, but always finitedimensional in each weight.

We will consider $k$-algebras $A=S / I$, where $S=k\left[x_{1}, \ldots, x_{n}\right]$ and $I$ is a homogeneous ideal with respect to both parity and weight. To such a $k$-algebra $A$, we will define a $\mathbb{Z}_{2} \times \mathbb{N}^{+} \times \mathbb{N}^{+}$-graded Lie algebra $\mathfrak{g}_{A}$ - the homotopy Lie algebra of $A$. It may be defined by the property $U\left(\mathfrak{g}_{A}\right)=\operatorname{Ext}_{A}(k, k)$, but we will give a more explicit definition (and more suitable for our purposes) using the minimal model of $A($ cf [L̈̈ 94]).

The minimal model of $A$ is a differential algebra $(\bigwedge V, d)$, where $V$ is a $\mathbb{Z}_{2} \times \mathbb{N} \times$ $\mathbb{N}^{+}$-graded vector space and where the first degree is the parity, the second degree is the homological degree and the third degree is the weight. The differential $d$ is a derivation which changes parity, lowers the homological degree by one and preserves weight. Moreover $d$ maps $V$ to $\bigwedge^{\geqslant 2} V=V \wedge V+V \wedge V \wedge V+\cdots$. The component of $d$ mapping $V$ to $V \wedge V$ is called $d_{2}$ (its extension as a derivation also satisfies $\left.d_{2}^{2}=0\right)$.

The ring $A$ is considered as a differential algebra with zero differential and concentrated in homological degree zero. There is a surjective map $\epsilon:(\bigwedge V, d) \rightarrow A$ preserving all degrees and inducing an isomorphism in homology. This means that $H_{i}(\bigwedge V, d)=0$ for homological degrees $i>0$ and if $A=k\left[x_{1}, \ldots, x_{n}\right] / I$ then $V$ in homological degree zero has a basis $X_{1}, \ldots, X_{n}, \epsilon\left(X_{i}\right)=x_{i}$ and $\epsilon(\operatorname{im}(d))=I$. The construction of $(\bigwedge V, d)$ is a straightforward procedure of killing cycles in a minimal way (see [Lö 94] for more details).

The Lie algebra $\mathfrak{g}_{A}$ is defined by $(s V)^{*}$ as a vector space, where $s$ changes parity, raises homological degree by one and preserves weight. The Lie product on $(s V)^{*}$ is in principle defined as the dual of $d_{2}$. More precisely, it is defined as a map $\left[(s V)^{*},(s V)^{*}\right]_{\mathcal{F}} \rightarrow(s V)^{*}$ in the following way (cf. Proposition 2.2),

$$
\left[(s V)^{*},(s V)^{*}\right]_{\mathcal{F}} \stackrel{\phi}{\rightarrow}\left([V, V]_{\mathcal{F}}\right)^{\perp}=(V \wedge V)^{*} \stackrel{d_{2}^{*}}{\rightarrow} V^{*} \stackrel{s^{-1}}{\rightarrow}(s V)^{*} .
$$

This map preserves all degrees and it may be checked that it satisfies the graded Jacobi identity. In coordinates the Lie product may be given as follows ( $\mathrm{cf}$ [ $\mathbf{A v} \mathbf{8 4}$ ], [Ha 92]).

Suppose $\{x, y, z, \ldots\}$ is an ordered basis for $V$ and let $\{X, Y, Z, \ldots\}$ denote the 
basis for $(s V)^{*}$ dual to $\{s x, s y, s z, \ldots\}$. If $d_{2} x=\sum_{y \leqslant z} c_{x y z} y z$ then,

$$
\begin{array}{ll}
{[Z, Y]=-\sum_{x}(-1)^{|y|} c_{x y z} X} & \text { if } y<z \\
(Y)^{2}=-\sum_{x} c_{x y y} X & \text { if } Y \text { is odd. }
\end{array}
$$

Inside $\mathfrak{g}_{A}$ there is the Lie subalgebra $\eta_{A}$ generated by $\mathfrak{g}_{A}^{1,1}$ (homological degree $=$ weight $=1)$. It is proved in $[\mathbf{L o ̈ ~ 9 4}]$ that $\eta_{A}$ coincides with the diagonal subalgebra $\oplus_{p \geqslant 1}^{\infty} \mathfrak{g}_{A}^{p, p}$ and it is proved in $[\operatorname{Pr} \mathbf{7 0}],[\mathbf{L} \ddot{\mathbf{~ 8 6}}]$ that $\mathrm{U}\left(\eta_{A}\right)=A^{!}$.

Definition 3.1. Let $\mathfrak{g}_{A}$ be the homotopy Lie algebra of $A$ and $\eta_{A}$ the Lie subalgebra of $\mathfrak{g}_{A}$ generated by $\mathfrak{g}_{A}^{1,1}$. The Lie algebra $L=\left(\eta_{A}\right) \geqslant 2=\left[\eta_{A}, \eta_{A}\right]$ is called the "Lie algebra associated to $A$ ". It has two degrees, a parity and a weight $(\geqslant 2)$.

Let $L$ be the Lie algebra associated to $A$ and let $\operatorname{gr}(L)$ denote the graded associated Lie algebra obtained from the lower central series $L \supset[L, L] \supset[L,[L, L]] \supset \cdots$; i.e.,

$$
\operatorname{gr}(L)=(\operatorname{gr}(L))_{1} \oplus(\operatorname{gr}(L))_{2} \oplus \cdots=L /[L, L] \oplus[L, L] /[L,[L, L]] \oplus \cdots .
$$

Each $(\operatorname{gr}(L))_{i}$ has an induced grading from $L,(\operatorname{gr}(L))_{i}=\oplus_{j}(\operatorname{gr}(L))_{i}^{j}$. The following theorem relates $\operatorname{gr}(L)$ to $\operatorname{Tor}^{S}(A, k)$, which is the homology of the Koszul complex of $A$ if $x_{i}$ is even for all $i$. The theorem may be seen as a study of an edge in Avramov's spectral sequence ([Av 74]), which has as $E^{2}$-term $\operatorname{Tor}^{\operatorname{Tor}^{S}(A, k)}(k, k)$ and which converges to $\mathrm{U}\left(\mathfrak{g}_{A}^{\geqslant 2}\right)^{*}$. At this point we want to thank J.-E. Roos, who conjectured part one of the theorem below and encouraged us to supply a proof.

\section{The Koszul homology in terms of the homotopy Lie algebra}

Theorem 4.1. Let $A=S / I=k\left[x_{1}, \ldots, x_{n}\right] / I$, where $I \subset\left(x_{1}, \ldots, x_{n}\right)^{2}$, be a $\mathbb{Z}_{2} \times \mathbb{N}$-graded connected $k$-algebra, commutative in the graded sense by the first degree, which is called the "parity"; the second degree is called the "weight". Let $H=\oplus H_{i, j}=\oplus \operatorname{Tor}_{i, j}^{S}(A, k)$ (here $i$ is the homological degree and $j$ is the weight), which is considered as a graded algebra in the following way; $H=k \oplus H_{(1)} \oplus H_{(2)} \oplus \cdots$, where $H_{(i)}=\oplus_{j} H_{j-i, j}$. Furthermore parity and weight in $A$ defines a bigrading on each $H_{(i)}$ (which is compatible with the multiplication in $H$ ). Let $L$ be the Lie algebra associated to $A$ and $\operatorname{gr}(L)$ the graded associated Lie algebra with respect to the lower central series. Then $\operatorname{gr}(L)$ has, besides a parity, two gradings, $\operatorname{gr}(L)=$ $\oplus_{i \geqslant 1, j \geqslant 2}(\operatorname{gr}(L))_{i}^{j}$ (see above). The following natural isomorphisms hold, where in (ii) the operator s just changes the parity.

(i) The 1-2-algebra of $H$ is isomorphic to the Koszul dual of $\mathrm{U}(\operatorname{gr}(L))$; i.e., $\left(H^{!}\right)^{!} \cong(\mathrm{U}(\operatorname{gr}(L)))^{!}$

(ii) $H_{(1)} \cong s(L /[L, L])^{*}$

$($ iii $) \operatorname{ker}\left(H_{(1)} \wedge H_{(1)} \rightarrow H_{(2)}\right) \cong([L, L] /[L,[L, L]])^{*}$ 
(iv)If $A$ is Koszul, then $H \cong \operatorname{Ext}_{\mathrm{U}(L)}(k, k)$ as algebras, in particular

$$
H_{j-i, j}=H_{(i), j} \cong\left(\operatorname{Ext}_{\mathrm{U}(L)}^{i}(k, k)\right)_{j} .
$$

Corollary 4.2. With notation as in the theorem, suppose $x_{i}$ is even for all $i$. Then

(i) $L^{j}=[L, L]^{j}$ for $j \geqslant n+2$

(ii)If $A$ is Koszul, then $\operatorname{gldim}(\mathrm{U}(L))<\infty$.

Corollary 4.3. With notation as in the theorem, we have

$$
\begin{aligned}
& (i) \operatorname{dim}_{k}\left(H_{1,2}\right)_{\text {even }}=\operatorname{dim}_{k}\left(L^{2}\right)_{\text {odd }} \\
& (\text { ii }) \operatorname{dim}_{k}\left(H_{1,2}\right)_{\text {odd }}=\operatorname{dim}_{k}\left(L^{2}\right)_{\text {even }} \\
& (\text { iii }) \operatorname{dim}_{k} H_{2,3}=\operatorname{dim}_{k} L^{3} \\
& (\text { iv }) \operatorname{dim}_{k} H_{3,4}=\operatorname{dim}_{k} L^{4} /\left[L^{2}, L^{2}\right] \\
& (\text { v }) \operatorname{dim}_{k}\left(\operatorname{ker}\left(H_{1,2} \wedge H_{1,2} \rightarrow H_{2,4}\right)\right)=\operatorname{dim}_{k}\left[L^{2}, L^{2}\right] \\
& (\text { vi }) \operatorname{dim}_{k}\left(\operatorname{ker}\left(H_{1,2} \otimes H_{2,3} \rightarrow H_{3,5}\right)\right)_{\text {even }}=\operatorname{dim}_{k}\left(\left[L^{2}, L^{3}\right]\right)_{\text {even }} \\
& (\text { vii }) \operatorname{dim}_{k}\left(\operatorname{ker}\left(H_{1,2} \otimes H_{2,3} \rightarrow H_{3,5}\right)\right)_{\text {odd }}=\operatorname{dim}_{k}\left(\left[L^{2}, L^{3}\right]\right)_{\text {odd }} \\
& \text { (viii }) \operatorname{dim}_{k} \operatorname{ker}\left(H_{2,3} \wedge H_{2,3} \oplus H_{1,2} \otimes H_{3,4} \rightarrow H_{4,6}\right)= \\
& \quad \operatorname{dim}_{k}\left(\left[L^{3}, L^{3}\right]+\left[L^{2}, L^{4}\right]\right) /\left[L^{2},\left[L^{2}, L^{2}\right]\right] .
\end{aligned}
$$

Remark 4.4. It has been known since long that $\operatorname{dim}_{k} H_{2,3}=\operatorname{dim}_{k} L^{3}$ and this was the starting point for our study of generic points and it is mainly this fact we will use in the applications of the theorem.

Proof of Theorem 4.1. We will give three different proofs. Even if the second one is short, we have included the other two, since we believe the methods used there are interesting in themselves. The first two use the minimal model of $A$. The third uses the cobar construction of (the dual of) the Koszul complex and the perturbation theory developed by Gugenheim, Stasheff, Lambe and others [Gu-La-St 91], [Hu-Ka 91], [Jo-La 00].

First proof. Let $(\bigwedge(V), d)$ be a minimal model of $A$ as described above. In particular this is a free resolution of $A$ as a module over $S \cong \bigwedge\left(V_{0}\right)$. Hence $\operatorname{Tor}^{S}(A, k)$ may be computed as the homology of $\bigwedge(V) \otimes_{S} k=\bigwedge(V \geqslant 1)$ with the induced differential $\bar{d}$. Due to the minimality of $(\bigwedge(V), d)$ and the fact that the generators in $S$ have positive weight, we may conclude that the weight is always greater than the homological degree. Let $W$ be the subspace of $V_{\geqslant 1}$ defined by elements of minimal possible weight; i.e., $W=\oplus_{i \geqslant 1} V_{i, i+1}$. Then $L=(s W)^{*}$. By degree reasons, the differential $\bar{d}$ maps $W$ to $W \wedge W$ and we will call this map $d_{2}$. Hence $\left(\bigwedge(W), d_{2}\right)$ is a subcomplex of $\left(\bigwedge\left(V_{\geqslant 1}\right), \bar{d}\right)$ and moreover $(\bar{d})^{-1}(W \wedge W)=W$. Put $W^{(1)}=\operatorname{ker}\left(d_{2}\right)$ and $W^{(2)}=d_{2}^{-1}\left(W^{(1)} \wedge W^{(1)}\right)$. Then $W^{(1)} \subset W^{(2)} \subset W$. We have

$$
\begin{aligned}
H_{(1)} & =W^{(1)} \\
\operatorname{ker}\left(H_{(1)} \wedge H_{(1)} \rightarrow H_{(2)}\right) & =d_{2}\left(W^{(2)}\right) .
\end{aligned}
$$

Claim.

$$
\left(s W^{(1)}\right)^{\perp}=[L, L]
$$




$$
\left(s W^{(2)}\right)^{\perp}=[L,[L, L]]
$$

Once this is proved, we get $\left(s H_{(1)}\right)^{*}=\left(s W^{(1)}\right)^{*}=(s W)^{*} /\left(s W^{(1)}\right)^{\perp}=L /[L, L]$ which proves $(i i)$. We also get $\left(s W^{(2)}\right)^{*}=L /[L,[L, L]]$. Let $d_{22}$ denote the restriction of $d_{2}$ to $W^{(2)}$, then $d_{22}: W^{(2)} \rightarrow W^{(1)} \wedge W^{(1)}$. By the above $\operatorname{ker}\left(H_{(1)} \wedge H_{(1)} \rightarrow H_{(2)}\right)=$ $\operatorname{im}\left(d_{22}\right)$. To prove $(i)$ we have to prove that $\phi^{-1}\left(\operatorname{im}\left(d_{22}\right)\right)^{\perp}$ is equal to the kernel of

$$
[L /[L, L], L /[L, L]]_{\mathcal{F}} \rightarrow[L, L] /[L,[L, L]] .
$$

But this is given as the kernel of the composition of the following maps.

$$
\left[\left(s W^{(1)}\right)^{*},\left(s W^{(1)}\right)^{*}\right]_{\mathcal{F}} \stackrel{\phi}{\rightarrow}\left(W^{(1)} \wedge W^{(1)}\right)^{*} \stackrel{d_{22}^{*}}{\longrightarrow}\left(W^{(2)}\right)^{*} \rightarrow\left(s W^{(2)}\right)^{*}
$$

Since the first and the last map are isomorphisms, this kernel is $\phi^{-1} \operatorname{ker}\left(d_{22}^{*}\right)=$ $\phi^{-1}\left(\operatorname{im}\left(d_{22}\right)\right)^{\perp}$ which proves $(i)$.

Now (iii) follows from Proposition $2.2(\mathrm{iv})$.

If $A$ is Koszul then $W=V_{\geqslant 1}$ and hence $\left(\bigwedge\left(V_{\geqslant 1}\right), \bar{d}\right)$ is the same as $\left(\bigwedge(W), d_{2}\right)$, which is the (generalized) standard Chevalley-Eilenberg complex (cf [Ch-Ei 48]) to compute the cohomology of the Lie algebra $(s W)^{*}$ (here the "wedge-degree" is the cohomological degree and the second degree of $W$ is the weight). Hence $H=\mathrm{H}\left(\left(\bigwedge(W), d_{2}\right)\right)=\operatorname{Ext}_{\mathrm{U}(L)}(k, k)$ which proves $(i v)$.

Proof of Claim.

We have that $[L, L]$ is the image of the map

$$
\left[(s W)^{*},(s W)^{*}\right]_{\mathcal{F}} \stackrel{\phi}{\rightarrow}(W \wedge W)^{*} \stackrel{d_{2}^{*}}{\rightarrow} W^{*} \rightarrow(s W)^{*} .
$$

Hence $[L, L]=s^{-1}\left(\operatorname{im}\left(d_{2}^{*}\right)\right)=s^{-1}\left(\operatorname{ker}\left(d_{2}\right)\right)^{\perp}=\left(s W^{(1)}\right)^{\perp}$.

To prove the second claim, we have to prove that the map above maps the subspace $\left[(s W)^{*},\left(s W^{(1)}\right)^{\perp}\right]_{\mathcal{F}}$ onto $\left(s W^{(2)}\right)^{\perp}$. We will do this in two steps

$$
\begin{aligned}
& \text { 1. } \phi\left(\left[(s W)^{*},\left(s W^{(1)}\right)^{\perp}\right]_{\mathcal{F}}\right)=\left(W^{(1)} \wedge W^{(1)}\right)^{\perp} \\
& \text { 2. } d_{2}^{*}\left(\left(W^{(1)} \wedge W^{(1)}\right)^{\perp}\right)=\left(W^{(2)}\right)^{\perp} .
\end{aligned}
$$

To prove 1., we first observe the following general fact. Suppose $B \rightarrow C$ is a surjective map with kernel $D$. Then $\mathrm{T}(B) /\langle D\rangle \cong \mathrm{T}(C)$ (use right exactness of the tensor product, diagram chasing and induction). Hence also $\mathcal{F}(B) /\langle D\rangle \cong \mathcal{F}(C)$. In particular $\operatorname{ker}\left([B, B]_{\mathcal{F}(B)} \rightarrow[C, C]_{\mathcal{F}(C)}\right)=[B, D]_{\mathcal{F}(B)}$. Now since $\phi$ is a natural isomorphism, $\phi$ induces an isomorphism

$$
\begin{aligned}
& \operatorname{ker}\left\{\left[(s W)^{*},(s W)^{*}\right]_{\mathcal{F}} \rightarrow\left[\left(s W^{(1)}\right)^{*},\left(s W^{(1)}\right)^{*}\right]_{\mathcal{F}}\right\} \rightarrow \\
& \operatorname{ker}\left\{(W \wedge W)^{*} \rightarrow\left(W^{(1)} \wedge W^{(1)}\right)^{*}\right\} .
\end{aligned}
$$

By the general fact above, the first kernel is $\left[(s W)^{*},\left(s W^{(1)}\right)^{\perp}\right]_{\mathcal{F}}$. Since the second kernel is $\left(W^{(1)} \wedge W^{(1)}\right)^{\perp}$, the first statement is proved. The second statement is a direct consequence of the following general fact (which is easily proven). Suppose $f: B \rightarrow C$ is a map of vector spaces and let $D$ be a subspace of $C$. Then $f^{*}\left(D^{\perp}\right)=$ $\left(f^{-1}(D)\right)^{\perp}$. 


\section{Second proof.}

We will use the same constructions and notations as in the first proof. Even if $A$ is not Koszul, we may argue in the same way as in the proof of $(i v)$ above to conclude that $\mathrm{H}\left(\bigwedge(W), d_{2}\right)=\operatorname{Ext}_{\mathrm{U}(L)}(k, k)$. Since $\left(\bigwedge(W), d_{2}\right)$ is a subcomplex of $\left(\bigwedge\left(V_{\geqslant 1}\right), \bar{d}\right)$ we get a map of algebras $\operatorname{Ext}_{\mathrm{U}(L)}(k, k) \rightarrow H$. We will study this map in low degrees and prove that the 1-2-algebra of $\operatorname{Ext}_{\mathrm{U}(L)}(k, k)$ is isomorphic to the 1-2-algebra of $H$. This, combined with Proposition 2.3 and Proposition 2.4, proves (i). Then $(i i)$ follows from $(i)$ and $(i i i)$ and $(i v)$ are proved as before.

Obviously we have $\operatorname{Ext}_{\mathrm{U}(L)}^{1}(k, k)=W^{(1)}=H_{(1)}$.

Since $(\bar{d})^{-1}(W \wedge W)=W$, we have

$$
\operatorname{im}\left(d_{2}\right) \cap\left(W^{(1)} \wedge W^{(1)}\right)=\operatorname{im}(\bar{d}) \cap\left(W^{(1)} \wedge W^{(1)}\right) .
$$

Hence,

$$
\begin{aligned}
\left(\operatorname{Ext}_{\mathrm{U}(L)}^{1}(k, k)\right)^{2} & =W^{(1)} \wedge W^{(1)} / \operatorname{im}\left(d_{2}\right) \cap\left(W^{(1)} \wedge W^{(1)}\right) \\
& =W^{(1)} \wedge W^{(1)} / \operatorname{im}(\bar{d}) \cap\left(W^{(1)} \wedge W^{(1)}\right)=\left(H_{(1)}\right)^{2} .
\end{aligned}
$$

It follows that

$$
\begin{aligned}
& \operatorname{ker}\left\{\operatorname{Ext}_{\mathrm{U}(L)}^{1}(k, k) \otimes \operatorname{Ext}_{\mathrm{U}(L)}^{1}(k, k) \rightarrow\left(\operatorname{Ext}_{\mathrm{U}(L)}^{1}(k, k)\right)^{2}\right\}= \\
& \operatorname{ker}\left\{H_{(1)} \otimes H_{(1)} \rightarrow\left(H_{(1)}\right)^{2}\right\}
\end{aligned}
$$

and hence the 1-2-algebra of $\operatorname{Ext}_{\mathrm{U}(L)}(k, k)$ is equal to the 1-2-algebra of $H$.

Third proof.

Let $(K, d)$ be the Koszul complex of $A$ with respect to $\left(x_{1}, \ldots, x_{n}\right)$; i.e., $(K, d)=$ $A\left[s x_{1}, \ldots, s x_{n} ; d\left(s x_{i}\right)=x_{i}\right]$ (we could also have used $\left(\bigwedge\left(V_{\geqslant 1}\right), d\right)$ from above). Then $(K, d)$ is a differential graded algebra (a DGA) and $\mathrm{H}(K, d)=H$. Since $k$ is a field, $H$ is a strong deformation retract of $(K, d)$ (an SDR); i.e., there are maps $f:(K, d) \rightarrow(H, 0)$ and $\nabla:(H, 0) \rightarrow(K, d)$ such that $f \nabla=i d$ and $\nabla f$ is homotopic to $i d$. From this it is possible to define an $\operatorname{SDR}\left(\mathrm{T}\left(\left(s K_{+}\right)^{*}\right), d_{K}\right) \rightarrow$ $\left(\mathrm{T}\left(\left(s H_{+}\right)^{*}\right), 0\right)$, where $d_{K}$ is the tensor extension of the differential on $K$. Now, the multiplication on $K_{+}$defines a differential $d_{2}$ on $\mathrm{T}\left(\left(s K_{+}\right)^{*}\right)$ in the same way as in Section 2; i.e., as the composition

$$
\left(s K_{+}\right)^{*} \rightarrow K_{+}^{*} \rightarrow\left(K_{+} \otimes K_{+}\right)^{*} \stackrel{\phi^{-1}}{\rightarrow}\left(s K_{+}\right)^{*} \otimes\left(s K_{+}\right)^{*}
$$

and $\left(\mathrm{T}\left(\left(s K_{+}\right)^{*}\right), d_{K}+d_{2}\right)$ is the cobar construction on $K^{*}$. Thinking of $d_{K}+d_{2}$ as a perturbation of $d_{K}$, one may use the perturbation lemma to obtain a differential $d$ on $\mathrm{T}\left(\left(s H_{+}\right)^{*}\right)$, such that $\left(\mathrm{T}\left(\left(s H_{+}\right)^{*}\right), d\right)$ is an SDR of $\left(\mathrm{T}\left(\left(s K_{+}\right)^{*}\right), d_{K}+d_{2}\right)$. Moreover $d$ is a derivation (see [Jo-La 00] ). But the cohomology of the cobar construction on $K^{*}$ is $\operatorname{Ext}_{(K, d)}(k, k)$ and this is $\mathrm{U}\left(\mathfrak{g}_{A}^{\geqslant 2}\right)([\mathbf{A v} \mathbf{7 4}])$.

Hence we have a DGA, $\left(\mathrm{T}\left(\left(s H_{+}\right)^{*}\right), d\right)$, with cohomology $\mathrm{U}\left(\mathfrak{g}_{A}^{\geqslant 2}\right)$, which is approximated by $\left(\mathrm{T}\left(\left(s H_{+}\right)^{*}\right), d_{2}\right)=\operatorname{Ext}_{H}(k, k)$. This is the "SDR-version" of Avramov's spectral sequence. (Compare this with the model $\left(\bigwedge\left(V_{\geqslant 1}\right), d\right)$ above, which has homology $H$, approximated by $\mathrm{H}\left(\bigwedge\left(V_{\geqslant 1}\right), d_{2}\right)=\operatorname{Ext}_{\mathrm{U}_{\left(\mathfrak{g}_{A}^{2}\right)}}(k, k)$.)

Put $V^{i, j}=\left(s H_{i-1, j}\right)^{*}$ for $i>1$ and put $V=\oplus V^{i, j}$. Then $d$ is determined by the value of $d$ on $V, d=d_{2}+d_{3}+\cdots$, where $d_{n}: V \rightarrow V^{\otimes n}, d_{2}$ is (in principal) the 
dual of the multiplication on $H$ and the higher $d_{n}$ are duals of Massey products. We have $V^{i, j}=0$ if $i>j$ and hence the elements of $V^{i, i}$ are cycles. The cohomology of $(\mathrm{T}(V), d)$ in degree $(i, i)$ is $\mathrm{U}(L)^{i}$ and hence

$$
\mathrm{U}(L)^{i}=V^{i, i} \oplus(V \otimes V)^{i, i} / \text { boundaries } \oplus(V \otimes V \otimes V)^{i, i} / \text { boundaries } \oplus \cdots
$$

This decomposition corresponds to the filtration of $\mathrm{U}(L)$ with respect to powers of $I=\mathrm{U}(L)^{+}$. This is so, because all elements of $(V \otimes V \otimes \cdots \otimes V)^{i, i}$ /boundaries are products of elements from $V^{j, j}$. In particular, $I / I^{2}=\oplus_{i} V^{i, i}=\left(s H_{(1)}\right)^{*}, I^{2} / I^{3}=$ $\oplus_{i}\left(V \otimes V / d_{2}(V)\right)^{i, i}$ and

$$
\operatorname{ker}\left(I / I^{2} \otimes I / I^{2} \rightarrow I^{2} / I^{3}\right)=\operatorname{im}\left(d_{2}\right)=\phi^{-1}\left(\operatorname{ker}\left(H_{(1)} \otimes H_{(1)} \rightarrow H_{(2)}\right)\right)^{\perp} .
$$

Hence the 1-2-algebra of $H$ is isomorphic to the Koszul dual of $\operatorname{gr}(\mathrm{U}(L))$. Thus, using Proposition 2.4, we get $(i)$ and $(i i)$; (iii) follows as before.

Suppose $A$ is Koszul. Then $(\mathrm{T}(V), d)$ has cohomology only on the diagonal. Consider $j-i$ as the homological degree of $\mathrm{T}(V)^{i, j}$. We have a map $(\mathrm{T}(V), d) \rightarrow$ $(\mathrm{U}(L), 0)$ (where $\mathrm{U}(L)$ is considered to be concentrated in homological degree zero), which induces an isomorphism in homology.

Hence $(\mathrm{T}(V), d)$ is a minimal (free associative) model of $\mathrm{U}(L)$. Such a model may always be constructed for any $\mathbb{Z}_{2} \times \mathbb{N}$-graded connected $k$-algebra $B$. This is similar to the construction of models $(\bigwedge(V), d)$ of commutative algebras described above, but now the space $(s V)^{*}$ is the algebra $\operatorname{Ext}_{B}^{+}(k, k)$ and the dual of $d_{2}$ (with the usual sign corrections) is the dual of the Yoneda product. Hence in our case we get $\operatorname{Ext}_{\mathrm{U}(L)}(k, k) \cong H$, which proves $(i v)$.

Problem 4.5. Suppose $A$ is generated by $A_{1}$. Is the converse of $(i v)$ in Theorem 4.1 true? Or, perhaps weaker, if the double Poincaré series of $\mathrm{U}(L), P_{\mathrm{U}(L)}(x, y)$, is equal to $H(1 / x, x y)$, is it then true that $A$ is Koszul?

Observe that the equality $P_{\mathrm{U}(L)}(x, y)=H(1 / x, x y)$ implies the equality $A(z) \cdot A^{!}(-z)=1$.

Added in proof: This problem has now been partially solved by Leonid Positselski (personal communication).

\section{The coordinate ring of generic points - IGC and MRC}

If $X=\left\{P_{1}, \ldots, P_{s}\right\}$ is a set of points in $P_{k}^{n}$, we denote the coordinate ring of $X, k\left[X_{0}, \ldots, X_{n}\right] / I(X)$, by $A_{X}$. It is well-known [Ge-Or 81] that the Hilbert series of $A_{X}$ satisfies $A_{X}(z) \leqslant \sum_{i \geqslant 0} \min \left(\left(\begin{array}{c}n+i \\ i\end{array}\right), s\right) z^{i}$. There is equality on an open non-empty set in $\left(P_{k}^{n}\right)^{s}$, and in case of equality the points are said to be in generic position. A point $P=\left(c_{0}: \cdots: c_{n}\right) \in P_{k}^{n}$ is called generic if the $c_{i}$ 's are algebraically independent over the prime field of $k$. A set of mutually generic points is of course in generic position. We will in the sequel always assume that our sets of points are generic. We may also assume that $s \geqslant n+1$, since otherwise the points lie in $P_{k}^{n-1}$.

Definition 5.1. Given $n$ and $s \geqslant n+1$, let $d \geqslant 2$ as a function of $n$ and $s$ be 
defined by

$$
\left(\begin{array}{c}
n+d-1 \\
d-1
\end{array}\right) \leqslant s<\left(\begin{array}{c}
n+d \\
d
\end{array}\right)
$$

It is well-known that

$$
\operatorname{Tor}_{i, j}^{S}\left(A_{X}, k\right)=0 \quad \text { if } \quad j \neq i+d-1, i+d
$$

(here and in the sequel $S$ will always denote $k\left[X_{0}, \ldots, X_{n}\right]$ ). In particular the minimal generators for the ideal $I_{X}$ are of degrees $d$ and $d+1$. Also, it is well-known that if $s=\left(\begin{array}{c}n+d-1 \\ d-1\end{array}\right)$, then $\operatorname{Tor}_{i, j}^{S}\left(A_{X}, k\right)=0$ if $j \neq i+d-1\left(A_{X}\right.$ has a linear resolution over $S)$.

It has been conjectured that the map $\left(\left(I_{X}\right)_{d}\right)^{n+1} \rightarrow\left(I_{X}\right)_{d+1}$, defined by $\left(f_{0}, \ldots, f_{n}\right) \mapsto \sum_{i=0}^{n} X_{i} f_{i}$, is either injective or surjective. This is called the Ideal Generating Conjecture (IGC). It is equivalent to the fact that

$$
\operatorname{Tor}_{1, d+1}^{S}\left(A_{X}, k\right)=0 \quad \text { or } \quad \operatorname{Tor}_{2, d+1}^{S}\left(A_{X}, k\right)=0 .
$$

The generalization that for all $i$,

$$
\operatorname{Tor}_{i, d+i}^{S}\left(A_{X}, k\right)=0 \quad \text { or } \quad \operatorname{Tor}_{i+1, d+i}^{S}\left(A_{X}, k\right)=0
$$

is called the Minimal Resolution Conjecture (MRC).

The MRC is proved for $P^{2}$ [Ge-Ma 84]; for $P^{3}$ [Ba-Ge 86]; for $P^{4}$ [Wa 95], [La 97]; for $P^{n}, n+1 \leqslant s \leqslant n+4$ [Ge-Lo 89], [Ca-Ro-Va 91]; for $P^{n}, s=\left(\begin{array}{c}n+2 \\ 2\end{array}\right)-$ $n$ [Lo 93]; for $P^{n}, n \leqslant 9, s \leqslant 50$ except $(n, s)=(6,11),(7,12),(8,13)$ [Be-Kr 94]; and for $P^{n}, s>6^{n^{3} \log n}$ [Hi-Si 96]. There has some time been computational evidence for some counterexamples by Schreyer, later [Bo 94], and [Be-Kr 94], and now there is shown to exist, for each $n \geqslant 6, n \neq 9$, one $s$ which gives a counterexample to MRC [Ei-Po 99]. In [Ge-Gr-Ro 86, Theorems 4.7 and 5.8] IGC is proved in several cases, e.g. for $s \leqslant 1+n+n^{2} / 4$ and for $\left(\begin{array}{c}n+2 \\ 2\end{array}\right)-n \leqslant s<\left(\begin{array}{c}n+2 \\ 2\end{array}\right)$. In [Co-Tr-Va 97] it is proved that $A_{X}$ is even Koszul if $s \leqslant 1+n+n^{2} / 4$.

We will now state some well-known and frequently used facts about the Hilbert series of $A_{X}$. Let $h_{i, j}^{n, s}=\operatorname{dim}_{k} \operatorname{Tor}_{i, j}^{S}\left(A_{X}, k\right), h_{i, j}^{n, s}=h_{i, j}$ when $n$ and $s$ are considered to be fixed and $H(x, y)=\sum h_{i, j} x^{i} y^{j}$.

Proposition 5.2. For any given $n$ and $s$ we have

$$
\begin{aligned}
& \text { (i) } A_{X}(z)=\sum_{j=0}^{d-1}\left(\begin{array}{c}
n+j \\
j
\end{array}\right) z^{j}+\frac{s z^{d}}{1-z} \\
& (i i)(1-z)^{n+1} A_{X}(z)=1+\sum_{j=0}^{n}(-1)^{j+1}\left(\begin{array}{c}
n \\
j
\end{array}\right)\left\{\left(\begin{array}{c}
n+d \\
d
\end{array}\right) \frac{d}{d+j}-s\right\} z^{d+j} \\
& (\text { iii })(1-z)^{n+1} A_{X}(z)=H(-1, z) \\
& (i v) h_{j+1, d+j}-h_{j, d+j}=\left(\begin{array}{c}
n \\
j
\end{array}\right)\left\{\left(\begin{array}{c}
n+d \\
d
\end{array}\right) \frac{d}{d+j}-s\right\}, \quad 0 \leqslant j \leqslant n .
\end{aligned}
$$


Proof. We give for convenience a short proof of $(i i)$. It is enough to prove the identity

$$
(1-z)^{n+1} \sum_{j=0}^{d-1}\left(\begin{array}{c}
n+j \\
j
\end{array}\right) z^{j}=1+\sum_{j=0}^{n}(-1)^{j+1}\left(\begin{array}{c}
n \\
j
\end{array}\right)\left(\begin{array}{c}
n+d \\
d
\end{array}\right) \frac{d}{d+j} z^{d+j} .
$$

This can easily be done by proving that both sides have the same derivative. Here is another way to deduce the identity without knowing the right hand side beforehand. Call the left hand side $p(z)$. It is a polynomial of degree $n+d$, divisible by $(1-z)^{n+1}$, and it may also be written as

$$
1-(1-z)^{n+1} \sum_{j=d}^{\infty}\left(\begin{array}{c}
n+j \\
j
\end{array}\right) z^{j}
$$

which shows that $p(z)-1$ is divisible (as a polynomial) by $z^{d}$. It follows that $p^{\prime}(z)$ is divisible by $z^{d-1}$ and by $(1-z)^{n}$ and hence there is a constant $c$ such that $p^{\prime}(z)=c z^{d-1}(1-z)^{n}=c \sum_{j=0}^{n}(-1)^{j}\left(\begin{array}{c}n \\ j\end{array}\right) z^{j+d-1}$. Intergrating this one gets

$$
p(z)=1+c \sum_{j=0}^{n}(-1)^{j}\left(\begin{array}{l}
n \\
j
\end{array}\right) \frac{1}{d+j} z^{j+d} .
$$

But the highest coefficient in $p(z)$ is $(-1)^{n+1}\left(\begin{array}{c}n+d-1 \\ d-1\end{array}\right)$ and hence $c(-1)^{n} \frac{1}{d+n}=$ $(-1)^{n+1}\left(\begin{array}{c}n+d-1 \\ d-1\end{array}\right)$, which gives $c=-d\left(\begin{array}{c}n+d \\ d\end{array}\right)$.

Definition 5.3. Let $n$ and $s$ be given and $d$ defined by Definition 5.1. Then $m$, as a function of $n$ and $s$, is defined as the least integer such that

$$
\left(\begin{array}{c}
n+d \\
d
\end{array}\right) \frac{d}{d+m}-s \leqslant 0
$$

and $t$, as a function of $n$ and $s$, is defined by

$$
t=\left(\begin{array}{c}
n \\
m-1
\end{array}\right)\left\{\left(\begin{array}{c}
n+d \\
d
\end{array}\right) \frac{d}{d+m-1}-s\right\}
$$

It is easy to see that if $\operatorname{Tor}_{i, d+i-1}^{S}\left(A_{X}, k\right)=0$, we have $\operatorname{Tor}_{j, d+j-1}^{S}\left(A_{X}, k\right)=0$ for all $j \geqslant i$, and if $\operatorname{Tor}_{i, d+i}^{S}\left(A_{X}, k\right)=0$, we have $\operatorname{Tor}_{j, d+j}^{S}\left(A_{X}, k\right)=0$ for all $j \leqslant i$. From this and Proposition 5.2 the following well-known proposition is easily deduced.

Proposition 5.4. For any given $n$ and s the following are equivalent.

(i) MRC holds

(ii) $h_{j-1, j+d-1}=h_{j+1, j+d}=0$ for some $j$

(iii) $h_{m-1, m+d-1}=h_{m+1, m+d}=0$

$(i v) h_{j, j+d-1}=\left(\begin{array}{c}n \\ j-1\end{array}\right)\left\{\left(\begin{array}{c}n+d \\ d\end{array}\right) \frac{d}{d+j-1}-s\right\} \quad$ and

$h_{j+1, j+d}=0$ for some $j$

$(v) h_{m, m+d-1}=t \quad$ and $\quad h_{m+1, m+d}=0$ 
From Proposition 5.2 the following well-known characterization of IGC immediately follows.

Proposition 5.5. For any given $n$ and $s, I G C$ holds if and only if

$$
h_{2, d+1}=\max \left(n\left\{\left(\begin{array}{c}
n+d \\
d
\end{array}\right) \frac{d}{d+1}-s\right\}, 0\right) \text {. }
$$

It is possible to interprete MRC and IGC in terms of the homotopy Lie algebra of $A_{X}$ (if $d>2$ ) or the Lie algebra associated to $A_{X}$ (if $d=2$ ).

Theorem 5.6. Let $X$ be a set of s generic points in $P_{k}^{n}$ and let $A_{X}$ be the coordinate ring of $X$. Let $\mathfrak{g}$ be the homotopy Lie algebra of $A_{X}$ and let $L$ be the Lie algebra associated to $A_{X}$ (see Definition 3.1). Let $d, m$ and $t$ be the numbers defined from $n$ and $s$ by Definition 5.1 and 5.3. Then, if $d>2$, we have the following

(i) MRC holds $\Longleftrightarrow \operatorname{dim}_{k} \mathfrak{g}^{m+1, m+d-1}=t \quad$ and $\mathfrak{g}^{m+2, m+d}=0$

(ii)IGC holds $\Longleftrightarrow \operatorname{dim}_{k} \mathfrak{g}^{3, d+1}=\max \left(n\left\{\left(\begin{array}{c}n+d \\ d\end{array}\right) \frac{d}{d+1}-s\right\}, 0\right)$

If $d=2$, we have the following

$$
\begin{aligned}
& \text { (iii)MRC holds } \Longleftrightarrow \operatorname{dim}_{k}(L /[L, L])^{m+1}=t \text { and }(L /[L, L])^{m+2}=0 \\
& \text { (iv)IGC holds } \Longleftrightarrow \operatorname{dim}_{k} L^{3}=\max \left(n\left\{\frac{(n+2)(n+1)}{3}-s\right\}, 0\right)
\end{aligned}
$$

Proof. Suppose first that $d>2$. In the third proof of theorem 4.1 it was proved that $\mathrm{U}\left(\mathfrak{g}_{A}^{\geqslant 2}\right)$ is the cohomology of $(\mathrm{T}(V), d)$, where $V^{i j}=\left(s H_{i-1, j}\right)^{*}$ for $i>1$ (and zero otherwise). Moreover the image of the differential is contained in $\oplus_{n \geqslant 2} V^{\otimes n}$. In our case, we have that $V^{i j}=0$ if $j-i \neq d-2, d-1$. The differential maps $V^{i j}$ to $\mathrm{T}(V)^{i+1, j}$. Hence, if this is nonzero, $d-2$ or $d-3$ must be a sum of at least two numbers each of which is $d-1$ or $d-2$. This is impossible if $d>2$. Hence the differential is zero and $\mathrm{U}\left(\mathfrak{g}_{A}^{\geqslant 2}\right) \cong \mathrm{T}(V)$. In particular $\mathfrak{g}_{A}^{i+1, i+d-1} \cong\left(H_{i, i+d-1}\right)^{*}$ and $\mathfrak{g}_{A}^{i+1, i+d} \cong\left(H_{i, i+d}\right)^{*}$. Now the statements $(i)$ and $(i i)$ follow from Proposition 5.4 $(v)$ and Proposition 5.5.

Suppose that $d=2$. The statements $(i i i)$ and $(i v)$ follow from the same propositions as above and Theorem 4.1 (ii).

The next theorem shows how the numbers $h_{i, j}$ are changed when the number of points is increased. For a given $d, h_{i, i+d}$ increases with $s$, while $h_{i+1, i+d}$ decreases. Moreover the rate of change is bounded by $\left(\begin{array}{c}n \\ i\end{array}\right)$.

Theorem 5.7. Let $A_{X}$ be the coordinate ring of a set $X$ of s generic points in $P_{k}^{n}$ and let $h_{i, j}^{n, s}=\operatorname{dim}_{k} \operatorname{Tor}_{i, j}^{S}\left(A_{X}, k\right)$. Then for any $i$, we have

$$
\begin{aligned}
& h_{i, i+d}^{n, s^{\prime}} \leqslant h_{i, i+d}^{n, s} \leqslant h_{i, i+d}^{n, s^{\prime}}+\left(\begin{array}{c}
n \\
i
\end{array}\right)\left(s-s^{\prime}\right) \text { and } \\
& h_{i+1, i+d}^{n, s} \leqslant h_{i+1, i+d}^{n, s^{\prime}} \leqslant h_{i+1, i+d}^{n, s}+\left(\begin{array}{c}
n \\
i
\end{array}\right)\left(s-s^{\prime}\right)
\end{aligned}
$$


whenever $s$ and $s^{\prime}$ define the same $d$; i.e., whenever $\left(\begin{array}{c}n+d-1 \\ d-1\end{array}\right) \leqslant s^{\prime} \leqslant s \leqslant\left(\begin{array}{c}n+d \\ d\end{array}\right)$.

Proof. Let $Y=\left\{P_{1}, \ldots, P_{s}\right\}$ be a set of $s$ generic points and let $X=Y \backslash\left\{P_{s}\right\}$. After a coordinate change we may assume that $P_{s}=(1: 0: \cdots: 0)$. Let $K=I_{X} / I_{Y}$ be the kernel of the natural map $A_{Y} \rightarrow A_{X}$. We get $K(z)=A_{Y}(z)-A_{X}(z)=$ $z^{d} /(1-z)$, so $K_{r}$ is one-dimensional in each degree $r \geqslant d$. Let $f_{d}+I_{Y} \in K$, where $f_{d} \notin I_{Y}$ is homogeneous of degree $d$; i.e., $f_{d}\left(P_{j}\right)=0$ for $j=1, \ldots, s-1$ and $f_{d}\left(P_{s}\right) \neq 0$. Then $\left(X_{i} f_{d}\right)\left(P_{j}\right)=0$ for all $j$ and $i=1, \ldots, n$, and hence $X_{i} f_{d} \in I_{Y}$ for $i=1, \ldots, n$. Moreover $\left(X_{0}^{r} f_{d}\right)\left(P_{j}\right)=0$ for $j=1, \ldots, s-1$, and $\left(X_{0}^{r} f_{d}\right)\left(P_{s}\right)=$ $f_{d}\left(P_{s}\right) \neq 0$ for every $r \geqslant 0$. Thus $K_{r+d}$ is generated by $X_{0}^{r} f_{d}+I_{Y}$. It follows that $K$ is generated by $f_{d}$ as an $S$-module and that $K \cong S /\left(X_{1}, \ldots, X_{n}\right)[-d]$. We now consider the long exact $\operatorname{Tor}_{*, i+d}^{S}(*, k)$-sequence of $0 \longrightarrow K \longrightarrow A_{Y} \longrightarrow$ $A_{X} \longrightarrow 0$. Since $K \cong S /\left(X_{1}, \ldots, X_{n}\right)[-d]$, we have $\operatorname{Tor}_{j, i+d}^{S}(K, k)=0$ if $j \neq i$ and $\operatorname{Tor}_{i, i+d}^{S}(K, k)=k^{\left(\begin{array}{c}n \\ i\end{array}\right)}$, and we get an exact sequence

$$
\begin{gathered}
0 \longrightarrow \operatorname{Tor}_{i+1, i+d}^{S}\left(A_{Y}, k\right) \longrightarrow \operatorname{Tor}_{i+1, i+d}^{S}\left(A_{X}, k\right) \longrightarrow \operatorname{Tor}_{i, i+d}^{S}(K, k) \longrightarrow \\
\operatorname{Tor}_{i, i+d}^{S}\left(A_{Y}, k\right) \longrightarrow \operatorname{Tor}_{i, i+d}^{S}\left(A_{X}, k\right) \longrightarrow 0
\end{gathered}
$$

The rightmost part of this sequence gives $h_{i, i+d}^{n, s-1} \leqslant h_{i, i+d}^{n, s} \leqslant h_{i, i+d}^{n, s-1}+\left(\begin{array}{c}n \\ i\end{array}\right)$, and the leftmost part gives $h_{i+1, i+d}^{n, s} \leqslant h_{i+1, i+d}^{n, s-1} \leqslant h_{i+1, i+d}^{n, s}+\left(\begin{array}{c}n \\ i\end{array}\right)$. The statement now follows by induction.

Suppose that MRC holds for $s^{\prime}$ and $s^{\prime \prime}$ points in $P_{k}^{n}$. Then, using the previous theorem, we may in some cases conclude that MRC also holds for all $s$ between $s^{\prime}$ and $s^{\prime \prime}$.

Theorem 5.8. Suppose $M R C$ holds for $s^{\prime}$ and $s^{\prime \prime}$ points in $P_{k}^{n}$, where for some $d \geqslant 2$ and some $j, 1 \leqslant j \leqslant n$,

$$
\left(\begin{array}{c}
n+d \\
d
\end{array}\right) \frac{d}{d+j} \leqslant s^{\prime}<s^{\prime \prime} \leqslant\left(\begin{array}{c}
n+d \\
d
\end{array}\right) \frac{d}{d+j-1} .
$$

Then MRC holds for any $s$ points in $P_{k}^{n}$, where $s^{\prime} \leqslant s \leqslant s^{\prime \prime}$.

Proof. By Theorem 5.4, we have $h_{j+1, j+d}=0$ for $s^{\prime}$ points $\left(m=j\right.$ for $\left.s^{\prime}\right)$. Hence, by Theorem 5.7, $h_{j+1, j+d}=0$ is true also for $s$ points. Also by Theorem 5.4, we have $h_{j-1, j+d-1}=0$ for $s^{\prime \prime}$ points. This is true if $s^{\prime \prime}<\left(\begin{array}{c}n+d \\ d\end{array}\right) \frac{d}{d+j-1}$, since then $m=j$ for $s^{\prime \prime}$. If $s^{\prime \prime}=\left(\begin{array}{c}n+d \\ d\end{array}\right) \frac{d}{d+j-1}$, then by Proposition 5.2 for $s^{\prime \prime}$ points, $h_{j, d+j-1}-$ $h_{j-1, d+j-1}=0$ and since MRC holds we must have $h_{j, d+j-1}=h_{j-1, d+j-1}=0$. Hence, by Theorem 5.7, $h_{j-1, j+d-1}=0$ is true also for $s$ points. Hence for $s$ points we have $h_{j+1, j+d}=h_{j-1, j+d-1}=0$, and hence MRC holds for $s$ points.

Corollary 5.9. In order to prove $M R C$ for $s$ in the interval $\left(\begin{array}{c}n+d-1 \\ d-1\end{array}\right) \leqslant s<\left(\begin{array}{c}n+d \\ d\end{array}\right)$, it is enough to prove $M R C$ for $s_{j}=\left\lceil\left(\begin{array}{c}n+d \\ d\end{array}\right) \frac{d}{d+j}\right\rceil, j=1, \ldots, n-1$, and for $s_{j}^{\prime}=$ $\left\lfloor\left(\begin{array}{c}n+d \\ d\end{array}\right) \frac{d}{d+j}\right\rfloor$. In order to prove IGC in the same interval, it is enough to prove IGC for $s=s_{1}$ and $s=s_{1}^{\prime}$. 
Until now the results in this section hold for any $d$. However, from now on, we will specialize to the case $d=2$. Using Theorem 5.8 and a result by Anna Lorenzini we can prove MRC in the interval $\left[\left(\begin{array}{c}n+2 \\ 2\end{array}\right)-n,\left(\begin{array}{c}n+2 \\ 2\end{array}\right)\right]$ for all $n$. This also follows from the results in [Ge-Gr-Ro 86].

Corollary 5.10. If $\left(\begin{array}{c}n+2 \\ 2\end{array}\right)-n \leqslant s \leqslant\left(\begin{array}{c}n+2 \\ 2\end{array}\right)$ then MRC holds.

Proof. MRC is proved in [Lo 93] for $s=\left(\begin{array}{c}n+2 \\ 2\end{array}\right)-n$. Since MRC also holds for $s=\left(\begin{array}{c}n+2 \\ 2\end{array}\right)$ and $\left(\begin{array}{c}n+2 \\ 2\end{array}\right) \frac{2}{3} \leqslant\left(\begin{array}{c}n+2 \\ 2\end{array}\right)-n \leqslant\left(\begin{array}{c}n+2 \\ 2\end{array}\right)$ the statement follows from Theorem 5.8 .

The following theorem will be useful in the next section, where we present results of calculations in the Lie algebra associated to $A_{X}$.

Theorem 5.11. Let $X$ be a set of $s$ generic points in $P_{k}^{n}$ and let $L$ be the Lie algebra associated to the coordinate ring of $X$ (see Definition 3.1). Suppose $s<\left(\begin{array}{c}n+2 \\ 2\end{array}\right)$. We then have

(i) $\quad L^{3}=0 \Rightarrow M R C$ (and hence also IGC) holds for $s^{\prime}$ points in $P_{k}^{n}$, where $s \leqslant s^{\prime} \leqslant\left(\begin{array}{c}n+2 \\ 2\end{array}\right)$

(ii) $\operatorname{dim}_{k} L^{3}=n\left\{\frac{(n+2)(n+1)}{3}-s\right\} \Rightarrow$ IGC holds for $s^{\prime}$ points in $P_{k}^{n}$, where $s^{\prime} \leqslant s$

(iii) $\operatorname{dim}_{k} L^{3}=n\left\{\frac{(n+2)(n+1)}{3}-s\right\}$ and $L^{4}=\left[L^{2}, L^{2}\right] \Rightarrow$ MRC holds

Proof. Suppose $L^{3}=0$. By Corollary $4.3($ iii $)$, we get $h_{2,3}=0$. Since also $h_{0,2}=0$, MRC holds for $s$ points by Proposition 5.4 (ii). Now MRC also holds for $s^{\prime \prime}=\left(\begin{array}{c}n+2 \\ 2\end{array}\right)$ points. Hence, $(i)$ follows from Theorem 5.8.

Suppose $\operatorname{dim}_{k} L^{3}=n\left\{\frac{(n+2)(n+1)}{3}-s\right\}$. Then, by Proposition $5.2(i v)(d=2$ and $j=1$ ), we get $h_{2,3}-h_{1,3}=\operatorname{dim}_{k} L^{3}$. But $\operatorname{dim}_{k} L^{3}=h_{2,3}$ and hence $h_{1,3}=0$. By Theorem 5.7, $h_{1,3}=0$ is true for any $s^{\prime}$ points in $P_{k}^{n}$, where $n+1 \leqslant s^{\prime} \leqslant s$ and hence (ii) follows.

The last statement follows, since as above $h_{1,3}=0$ and, by Corollary $4.3(i v)$, $h_{3,4}=0$. Now use Proposition 5.4 (ii).

\section{Results of calculations}

We note that MRC is proved for $s<\left(\begin{array}{c}n+2 \\ 2\end{array}\right)$ if $n<9$, except for $(n, s)=$ $(6,11),(7,12),(8,13)$, and also for $s \leqslant 50$ if $n=9$, [Be-Kr 94]. Thus we treat only the case $n \geqslant 9$.

Theorem 6.1. For $n \leqslant 20$ and $(n+1)(n+2) / 3 \leqslant s<(n+1)(n+2) / 2$ MRC holds.

Proof. Since $s<(n+1)(n+2) / 2$ we have $d=2$. From the table in Section 11 we get that $L^{3}=0$ for $n \leqslant 20$ and $s=\lceil(n+1)(n+2) / 3\rceil$. Hence the result follows from Theorem $5.11(i)$. 
Theorem 6.2. If $n \leqslant 20$ and $s<(n+1)(n+2) / 2$, IGC holds.

Proof. If $s \geqslant(n+2)(n+1) / 3$ the conclusion follows from the previous theorem. From the table in Section 11 we get that $\operatorname{dim}_{k} L^{3}=n\left\{\frac{(n+2)(n+1)}{3}-s\right\}$ for $s=$ $\lfloor(n+1)(n+2) / 3\rfloor$. Hence the result follows from Theorem 5.9.

\section{Definition 6.3.}

$$
\begin{aligned}
& l_{3}=\lceil(n+1)(n+2) / 3\rceil \\
& l_{4}=\left\lceil n^{2}+n+1 / 2-\frac{1}{2} \sqrt{2 n^{4}-2 n^{2}+1}\right\rceil \\
& l_{5}=\left\lceil n^{2} / 2+n+1-\frac{1}{10} \sqrt{5 n^{4}+20}\right\rceil
\end{aligned}
$$

We conjecture that if $s \geqslant l_{i}$, then $L^{i}=0, i=3,4,5$, cf. Conjecture 8.1. From the table in Section 11, it follows that the conjecture is true for $L^{3}$ if $n \leqslant 20$, for $L^{4}$, if $n \leqslant 17$, and for $L^{5}$ if $n \leqslant 11$. We can use the result for $L^{4}$ to extend the interval where we can prove MRC.

Theorem 6.4. If $n \leqslant 17$ and $l_{4} \leqslant s \leqslant\left(\begin{array}{c}n+2 \\ 2\end{array}\right)$ then MRC holds.

Proof. From the table in Section 11 we get that $L^{4}=0$ for $s=l_{4}$. Hence by Theorem 5.7 it is true also for $s \geqslant l_{4}$. Corollary 4.3 gives $h_{3,4}=0$ for $s \geqslant l_{4}$. Since we have proved that $\operatorname{dim}_{k} L^{3}=n\left\{\frac{(n+2)(n+1)}{3}-s\right\}$ for $s=\lfloor(n+1)(n+2) / 3\rfloor$, it follows that $h_{1,3}=0$ for that many points and then, by Theorem 5.7, it follows that $h_{1,3}=0$ for $s \leqslant(n+1)(n+2) / 3$. Hence $h_{1,3}=h_{3,4}=0$ for $l_{4} \leqslant s \leqslant(n+1)(n+2) / 3$ and hence MRC holds in this interval. But by Theorem 6.1, MRC holds in the interval $l_{3} \leqslant s \leqslant\left(\begin{array}{c}n+2 \\ 2\end{array}\right)$.

Using Theorem 5.6 (iii), we can show MRC in a few more cases.

Theorem 6.5. MRC is true if

a) $n=9$ and $d=2$ (i.e., $s<55$ ).

b) $(n, s)=(10,39),(10,40),(11,47)$, and $(12,55)$.

Proof. For $n=9$ and $s \leqslant 50$, MRC is proved in [Be-Kr 94]. For $50<s<55$ we have proved that $L^{3}=0$ (see Section 11) and hence MRC holds by Theorem 5.11. For the cases in b) we have used the Mathematica program "liedim" to show that $\left[L^{2}, L^{2}\right]=L^{4}$. Then, since $\operatorname{dim}_{k} L^{3}=t$, the statement follows from Theorem 5.6. (In fact, the case $(10,40)$ may be derived from $(10,39)$ and $(10,43)$ using Theorem 5.8.)

It is natural to conjecture that

$$
\operatorname{Tor}_{r, s}^{S}\left(A_{X}, k\right) \wedge \operatorname{Tor}_{t, u}^{S}\left(A_{X}, k\right) \rightarrow \operatorname{Tor}_{r+t, s+u}^{S}\left(A_{X}, k\right)
$$

is either injective or surjective. We can prove this in some special cases. 
Theorem 6.6. Let $A_{X}$ be the coordinate ring of $s$ generic points in $P_{k}^{n}$ and let $S=k\left[x_{0}, \ldots, x_{n}\right]$. If $n \leqslant 17$ and $s \geqslant l_{4}$ the multiplication

$$
\operatorname{Tor}_{1,2}^{S}\left(A_{X}, k\right) \wedge \operatorname{Tor}_{1,2}^{S}\left(A_{X}, k\right) \rightarrow \operatorname{Tor}_{2,4}^{S}\left(A_{X}, k\right)
$$

is injective.

Proof. We have shown that $L^{4}=0$ in this interval (see Section 11). Thus Corollary $4.3(v)$ gives that the multiplication is injective.

Theorem 6.7. Let $A_{X}$ be the coordinate ring of $s$ generic points in $P_{k}^{n}$ and let $S=k\left[x_{0}, \ldots, x_{n}\right]$. For $(n, s)=$

$$
\begin{aligned}
& (3,6),(5,13),(6,17),(6,18),(7,21),(7,22),(8,26),(8,27),(9,32),(9,33), \\
& (10,39),(10,40),(11,47),(12,55)
\end{aligned}
$$

the multiplication

$$
\operatorname{Tor}_{1,2}^{S}\left(A_{X}, k\right) \wedge \operatorname{Tor}_{1,2}^{S}\left(A_{X}, k\right) \rightarrow \operatorname{Tor}_{2,4}^{S}\left(A_{X}, k\right)
$$

is surjective.

Proof. For these pairs of $(n, s)$, we have that $\operatorname{Tor}_{i, *}^{S}\left(A_{X}, k\right)$ for $i \leqslant 3$ have the expected dimensions for MRC ([Be-Kr 94] and Theorem 6.5). By Corollary $4.3(v)$, the kernel of the multiplication map in the theorem has dimension $\operatorname{dim}_{k}\left[L^{2}, L^{2}\right]$, and by Corollary $4.3(i v), h_{3,4}=\operatorname{dim}_{k} L^{4} /\left[L^{2}, L^{2}\right]$. Since MRC holds, we know $h_{1,2}, h_{2,4}$, and $h_{3,4}$. From Section 11 we know $\operatorname{dim}_{k} L^{4}$ for all pairs $(n, s)$ in the theorem. Thus the statement follows by computing dimensions. E.g., if $n=6$ and $s=17$ we have that $h_{1,2}=\operatorname{dim}_{k} L^{2}=11$ and hence $\operatorname{dim}_{k}\left(\operatorname{Tor}_{1,2}^{S}\left(A_{X}, k\right) \wedge \operatorname{Tor}_{1,2}^{S}\left(A_{X}, k\right)\right)=55$. Also, $h_{3,4}=0$ since MRC holds and $m=2$, and, by Proposition $5.2(\mathrm{iv})$, we get $h_{2,4}=45$. Hence by Corollary $4.3(i v), L^{4}=\left[L^{2}, L^{2}\right]$, and from the table, $\operatorname{dim}_{k} L^{4}=10$. Hence the map is surjective, since the kernel is 10-dimensional by Corollary $4.3(v)$.

We can check the conjecture about the multiplication in a few more cases. If $s=n+2$, it is easy to see that $A_{X}$ is Gorenstein. By reasons of degree, the only possible nonsurjective multiplication is $\operatorname{Tor}_{i} \wedge \operatorname{Tor}_{n-i} \rightarrow \operatorname{Tor}_{n}$. This is surjective, since $A_{X}$ is Gorenstein.

In $P^{3}$ the multiplication is injective or surjective for any choice of degrees and number of points. If $s \geqslant 7$ this follows from Theorem 6.6. If $s=5, A_{X}$ is Gorenstein. If $s=4$ the resolution is linear and the multiplication trivial. For the remaining case $s=6$, the multiplication

$$
\operatorname{Tor}_{1,2}^{S}\left(A_{X}, k\right) \wedge \operatorname{Tor}_{1,2}^{S}\left(A_{X}, k\right) \rightarrow \operatorname{Tor}_{2,4}^{S}\left(A_{X}, k\right)
$$

is surjective by Theorem 6.7. That the multiplication

$$
\operatorname{Tor}_{1,2}^{S}\left(A_{X}, k\right) \otimes \operatorname{Tor}_{2,3}^{S}\left(A_{X}, k\right) \rightarrow \operatorname{Tor}_{3,5}^{S}\left(A_{X}, k\right)
$$

is surjective follows in a similar way as in the proof of Theorem 6.7 by determining $\operatorname{dim}_{k} L^{5}$ (cf. Section 11). 


\section{Ideals generated by generic quadratic forms}

We call a quadratic form $\sum_{1 \leqslant i \leqslant j \leqslant n} c_{i j} x_{i} x_{j}$ in $k\left[x_{1}, \ldots, x_{n}\right]$ generic if $\left\{c_{i j}\right\}$ is algebraically independent over the prime field of $k$ (the variables $x_{i}$ are considered to be even). We will now consider ideals $\left(f_{1}, \ldots, f_{t}\right)$ in $k\left[x_{1}, \ldots, x_{n}\right]$, where $f_{k}=$ $\sum_{1 \leqslant i \leqslant j \leqslant n} c_{i j}^{(k)} x_{i} x_{j}$ and $\left\{c_{i j}^{(k)}\right\}$ is algebraically independent over the prime field of $k$. For simplicity we say that such an ideal is generated by $t$ generic quadratic forms. We will determine for which $n$ and $t$ we have that $k\left[x_{1}, \ldots, x_{n}\right] / I$ is a Koszul algebra if $I$ is generated by $t$ quadratic generic forms. We will identify an ideal $\left(g_{1}, \ldots, g_{t}\right)$ generated by linearly independent quadratic elements (and thus the quadratic algebra $\left.k\left[x_{1}, \ldots, x_{n}\right] /\left(g_{1}, \ldots, g_{t}\right)\right)$ with a point in $\operatorname{Grass}\left(\left(\begin{array}{c}n+1 \\ 2\end{array}\right), t\right)$. One part of the following theorem is proved in [Co-Tr-Va 97].

Theorem 7.1. Let $A=k\left[x_{1}, \ldots, x_{n}\right] /\left(g_{1}, \ldots, g_{t}\right)$ be a quadratic algebra, where the variables $x_{i}$ are considered to be even. If $t \leqslant n$ there is an open non-empty subset of $\operatorname{Grass}\left(\left(\begin{array}{c}n+1 \\ 2\end{array}\right), t\right)$ on which $A$ is Koszul. If $t \geqslant n^{2} / 4+n / 2$ there is a nonempty countable intersection of open sets in $\operatorname{Grass}\left(\left(\begin{array}{c}n+1 \\ 2\end{array}\right), t\right)$ on which $A$ is Koszul. If $n<t<n^{2} / 4+n / 2$, then $A$ is not Koszul on an open non-empty subset of $\operatorname{Grass}\left(\left(\begin{array}{c}n+1 \\ 2\end{array}\right), t\right)$. In particular, if $A=k\left[x_{1}, \ldots, x_{n}\right] / I$, where $I$ is generated by $t$ generic quadratic forms, then $A$ is Koszul if and only if $t \leqslant n$ or $t \geqslant n^{2} / 4+n / 2$.

Proof. If $t \leqslant n$, then almost all $A$ are complete intersections, and complete intersections are Koszul, cf. [Fr-Lö 91].

Now let $t \geqslant n^{2} / 4+n / 2$. Consider the rings $A$ with $t$ fixed as points in $G=$ $\operatorname{Grass}\left(\left(\begin{array}{c}n+1 \\ 2\end{array}\right), t\right)$. The subset of $G$ corresponding to rings $A$ with $A_{3}=0$ is open. For such $A$, the Bar complex of $k$ over $A$ is based on the vector space $A_{1} \oplus A_{2}$, which has the fixed dimension $n+\left(\begin{array}{c}n+1 \\ 2\end{array}\right)-t$, and the differential is expressed by means of the multiplication in $A$. Hence, it is an open condition that the homology is zero in a certain bidegree $(i, j)$, and hence $A_{3}=0$ and $\operatorname{Tor}_{i, j}^{A}(k, k)=0$ is an open condition for fixed $(i, j)$. That $A$ is Koszul, i.e., $\operatorname{Tor}_{i, j}^{A}(k, k)=0$ if $i \neq j$, is therefore true on an intersection of open sets. It suffices to show that this intersection is non-empty. If $A=k\left[x_{1}, \ldots, x_{a}\right] /\left(x_{1}, \ldots, x_{a}\right)^{2} \otimes k\left[x_{1}, \ldots, x_{b}\right] /\left(x_{1}, \ldots, x_{b}\right)^{2}$, with $a=\lceil n / 2\rceil$ and $b=\lfloor n / 2\rfloor$, we have an example of a Koszul algebra in case $t=\left\lceil n^{2} / 4+n / 2\right\rceil$, [Fr 75]. If $t$ is larger we could just divide by some more quadratic monomials.

Now let $n<t \leqslant\left(\begin{array}{c}n+2 \\ 3\end{array}\right) / n$. Then, by [Ho-La 87], there is a non-empty subset of $G$ with $\left\{x_{i} g_{j}\right\}$ linearly independent, where $I=\left(g_{1}, \ldots, g_{t}\right)$. By Corollary 4.3 (iii), this gives $L^{3}=0$. If $A$ were Koszul, then $U\left(\eta_{A}\right)$ is equal to $\operatorname{Ext}_{\mathrm{A}}(k, k)$. Then $A$ would be a complete intersection by [Gu 71], a contradiction since $t>n$.

Finally let $\left(\begin{array}{c}n+2 \\ 3\end{array}\right) / n \leqslant t<n^{2} / 4+n / 2$. Then, by [Ho-La 87], $A_{3}=0$ on a nonempty open subset of $G$. If $A$ is Koszul, then $A^{!}(z)=1 /\left(1-n z+\left(\left(\begin{array}{c}n+1 \\ 2\end{array}\right)-t\right) z^{2}\right)=$ $1 /\left((1-n z / 2)^{2}+\left(n^{2} / 4+n / 2-t\right) z^{2}\right)$. Hence $A^{!}(z)$ has no pole on the real axis. But then the coefficients in $A^{!}(z)$ are not all non-negative, since a series with non-negative coefficients has a singularity on the positive real axis if its radius of convergence is finite.

Remark 7.2. It follows from the theorem above that the "smallest" algebra defined by generic quadratic forms which is not Koszul is obtained by choosing $n=4$ and 
$t=5$. This was in fact the first example of a non-Koszul quadratic algebra found by Christer Lech 1975.

To state some conjectures about the coordinate ring of generic quadratic forms, we need a concept from non-commutative graded algebras.

Let $B$ be an $\mathbb{N}$-graded associative algebra and $\left\{y_{1}, \ldots, y_{r}\right\}$ a set of homogeneous elements in $B$ of degrees $d_{1}, \ldots, d_{r}$, respectively. Then

$$
B /\left(y_{1}, \ldots, y_{r}\right)(z) \geqslant B(z) /\left(1+\left(z^{d_{1}}+\cdots+z^{d_{r}}\right) B(z)\right)
$$

and the set is called strongly free in $B$ if there is equality, [An 82]. Any subset of a strongly free set is strongly free. If $\left\{y_{1}, \ldots, y_{r}\right\}$ is a strongly free set of quadratic elements in the free associative algebra $k\left\langle X_{1}, \ldots, X_{n}\right\rangle$, we get

$$
k\left\langle X_{1}, \ldots, X_{n}\right\rangle /\left(y_{1}, \ldots, y_{r}\right)(z)=1 /\left(1-n z+r z^{2}\right) .
$$

Such an algebra will be called a non-commutative c.i. (complete intersection). They play much the same rôle for non-commutative algebras as complete intersections do for commutative algebras. E.g., the algebra

$B=k\left\langle X_{1}, \ldots, X_{n}\right\rangle /\left(y_{1}, \ldots, y_{r}\right)$ is a non-commutative c.i. if and only if $k\left\langle X_{1}, \ldots, X_{n}, Y_{1}, \ldots Y_{r} ; d X_{i}=0, d Y_{i}=y_{i}\right\rangle$ is a minimal model of $B$ (cf. end of proof of Theorem 4.1). It follows that $B$ is a non-commutative c.i. if and only if $\operatorname{gldim}(B) \leqslant 2$, and hence a non-commutative c.i. is Koszul. Also, for a quadratic algebra $A, A^{!}$is a non-commutative c.i. if and only if $A$ is Koszul and $A_{3}=0$.

We will now consider ideals $\left(h_{1}, \ldots, h_{r}\right)$ in $k\left\langle X_{1}, \ldots, X_{n}\right\rangle$, where the variables $X_{i}$ are odd and where $h_{k}=\sum_{1 \leqslant i \leqslant j \leqslant n} d_{i j}^{(k)}\left[X_{i}, X_{j}\right]$ and $\left[X_{i}, X_{j}\right]=X_{i} X_{j}+X_{j} X_{i}$. Such an element is called a Hopf element and the corresponding quotient algebra a quadratic Hopf algebra. We will identify an ideal $\left(h_{1}, \ldots, h_{r}\right)$ generated by linearly independent quadratic Hopf elements (and thus also the quadratic Hopf algebra $\left.k\left\langle X_{1}, \ldots, X_{n}\right\rangle /\left(h_{1}, \ldots, h_{r}\right)\right)$ with a point in $\operatorname{Grass}\left(\left(\begin{array}{c}n+1 \\ 2\end{array}\right), r\right)$. As before we will say that the ideal $\left(h_{1}, \ldots, h_{r}\right)$ is generated by $r$ generic quadratic Hopf elements if the coefficients $\left\{d_{i j}^{(k)}\right\}$ is algebraically independent over the prime field of $k$.

There is a well-known duality between Grassmanians yielding the following proposition.

Proposition 7.3. The correspondence $A \leftrightarrow A^{!}$defines an isomorphism of projective manifolds between $\operatorname{Grass}\left(\left(\begin{array}{c}n+1 \\ 2\end{array}\right), t\right)$ and $\operatorname{Grass}\left(\left(\begin{array}{c}n+1 \\ 2\end{array}\right),\left(\begin{array}{c}n+1 \\ 2\end{array}\right)-t\right)$

Proof. The relations in $A^{!}$are obtained from those in $A$ by solving a system of linear equations. By Cramer's rule the solutions may be expressed as rational functions and locally the same determinant may be used in the denominators.

Now the dual version of Theorem 7.1 directly follows.

Theorem 7.4. Let $B=k\left\langle X_{1}, \ldots, X_{n}\right\rangle /\left(h_{1}, \ldots, h_{r}\right)$ be a quadratic Hopf algebra, where the variables $X_{i}$ are considered to be odd. If $r \leqslant n^{2} / 4$ there is a countable intersection of open sets in $\operatorname{Grass}\left(\left(\begin{array}{c}n+1 \\ 2\end{array}\right), r\right)$ on which B is Koszul. If $r \geqslant\left(\begin{array}{l}n \\ 2\end{array}\right)$ there is an open non-empty subset of $\operatorname{Grass}\left(\left(\begin{array}{c}n+1 \\ 2\end{array}\right), r\right)$ on which $B$ is Koszul. If $n^{2} / 4<$ 
$r<\left(\begin{array}{c}n \\ 2\end{array}\right)$, then $A$ is not Koszul on an open non-empty subset of $\operatorname{Grass}\left(\left(\begin{array}{c}n+1 \\ 2\end{array}\right), r\right)$. In particular, if $B=k\left\langle X_{1}, \ldots, X_{n}\right\rangle / J$, where $J$ is generated by $r$ generic quadratic Hopf elements, then $B$ is Koszul if and only if $r \leqslant n^{2} / 4$ or $r \geqslant\left(\begin{array}{l}n \\ 2\end{array}\right)$ and $B$ is a non-commutative c.i. if and only if $r \leqslant n^{2} / 4$.

Proof. To get the statement about the Koszul property, we just use Theorem 7.1 and Proposition 7.3, observing that $\left(\begin{array}{c}n+1 \\ 2\end{array}\right)-n^{2} / 4-n / 2=n^{2} / 4$ and $\left(\begin{array}{c}n+1 \\ 2\end{array}\right)-n=\left(\begin{array}{c}n \\ 2\end{array}\right)$. The last statement (proved in [An 82]) follows from the remark above that $B$ is a non-commutative c.i. if and only if $B^{!}$is Koszul and $B_{3}^{!}=0$, which by the proof of Theorem 7.1 is true iff $\left(\begin{array}{c}n+1 \\ 2\end{array}\right)-r \geqslant(n+2)(n+1) / 6$ and also $\left(\begin{array}{c}n+1 \\ 2\end{array}\right)-r \geqslant n^{2} / 4+n / 2$ or $\left(\begin{array}{c}n+1 \\ 2\end{array}\right)-r \leqslant n$. But for $n \geqslant 2$ we have $n \leqslant(n+2)(n+1) / 6 \leqslant n^{2} / 4+n / 2$.

Conjecture 7.5. Let $A=k\left[x_{1}, \ldots, x_{n}\right] / I$, where the variables $x_{i}$ are even and where $I$ is generated by quadratic generic elements. Then either $A^{!}$is a non-commutative c.i. or $\eta_{A}$ is nilpotent, where $A^{!}=U\left(\eta_{A}\right)$.

For a formal power series $\sum_{i \geqslant 0} a_{i} z^{i}$ we let $\left(\sum_{i \geqslant 0} a_{i} z^{i}\right)_{+}=\sum_{i \geqslant 0} b_{i} z^{i}$, where $b_{i}=a_{i}$ if $a_{j}>0$ for all $j \leqslant i$, and $b_{i}=0$ otherwise. A formal power series $p(z)$ with integer coefficients and constant term 1 may be written in the form $p(z)=$ $\prod_{k=1}^{\infty}\left(1+z^{2 k-1}\right)^{e_{2 k-1}} /\left(1-z^{2 k}\right)^{e_{2 k}}$. We denote by $\log (p(z))$ the series $\sum_{i \geqslant 1} e_{i} z^{i}$. Recall that, for a graded algebra $A$, we have $U\left(\eta_{A}\right)=A^{!}$, so $\eta_{A}(z)=\log \left(A^{!}(z)\right)$.

We now make the conjecture more precise.

Conjecture 7.6. Let $A=k\left[x_{1}, \ldots, x_{n}\right] / I$, where the variables $x_{i}$ are even and where $I$ is generated by $\left(\begin{array}{c}n+1 \\ 2\end{array}\right)-r$ quadratic generic forms. Then $\eta_{A}(z)=(\log (1 /(1-$ $\left.\left.\left.n z+r z^{2}\right)\right)\right)_{+}$.

Theorem 7.7. Conjecture 7.6 is true in the following cases

a) If $A$ is Koszul.

b) If $n \leqslant 8$.

c) If $r \geqslant\left(n^{2}-1\right) / 3$ and $n \leqslant 20$.

d) If $r \geqslant n^{2}-1 / 2-\frac{1}{2} \sqrt{2 n^{4}-2 n^{2}+1}$ and $n \leqslant 17$.

e) If $r \geqslant n^{2} / 2-\frac{1}{10} \sqrt{5 n^{4}+20}$ and $n \leqslant 10$.

f) If $(n, r)=(9,22)$.

Proof. a) According to Theorem 7.1, $A$ is Koszul if and only if $r \geqslant\left(\begin{array}{l}n \\ 2\end{array}\right)$ or $r \leqslant n^{2} / 4$. In the first case we know that $A$ is a complete intersection, so $\eta_{A}(z)=n z+\left(\left(\begin{array}{c}n+1 \\ 2\end{array}\right)-\right.$ $r) z^{2}$. A simple calculation shows that $\left(\log \left(1 /\left(1-n z+r z^{2}\right)\right)\right)_{+}=n z+\left(\left(\begin{array}{c}n+1 \\ 2\end{array}\right)-r\right) z^{2}$ if and only if $r \geqslant\left(n^{2}-1\right) / 3$. Since $\left(\begin{array}{l}n \\ 2\end{array}\right) \geqslant\left(n^{2}-1\right) / 3$, the result follows in the first case. In the second case, $A^{!}$is a non-commutative c.i. (since $A$ is Koszul and $\left.A_{3}=0\right)$ and hence $A^{!}(z)=1 /\left(1-n z+r z^{2}\right)$. It follows that $\eta_{A}(z)=\log \left(A^{!}(z)\right)=$ $\log \left(1 /\left(1-n z+r z^{2}\right)\right)$. Hence also $\eta_{A}(z)=\left(\log \left(1 /\left(1-n z+r z^{2}\right)\right)\right)_{+}$, since $\left(\eta_{A}\right)_{i}=0$ for some $i$ implies that $\left(\eta_{A}\right)_{j}=0$ for all $j \geqslant i$.

b) We show how the result follows for $n=8$. Then $A$ is Koszul if $\left(\begin{array}{l}9 \\ 2\end{array}\right)-r=t \geqslant$ $8^{2} / 4+8 / 2=20$; i.e., if $A$ has at least 20 quadratic relations. If $A$ has $t$ quadratic relations, then $A^{!}$has $r=\left(\begin{array}{l}9 \\ 2\end{array}\right)-t=36-t$ quadratic relations, so $A^{!}(z) \geqslant 1 /(1-$ $\left.8 z+r z^{2}\right)$ and hence $\eta_{A}(z) \geqslant\left(\log \left(1 /\left(1-8 z+r z^{2}\right)\right)\right)_{+}$. To see that there is equality 
generically when $t<20$, it is sufficient to produce one example. Let $\bar{A}=A_{X} / y$, where $A_{X}$ is the coordinate ring of $s$ points in $P^{8}$, and $y$ is a linear nonzerodivisor in $A_{X}$. Then $A_{X}$ and $A$ have $\left(\begin{array}{c}10 \\ 2\end{array}\right)-s=45-s$ quadratic relations. Thus we can use our calculations for 26 or more points in $P^{8}$. For 26 to 30 points we verify the conjecture directly from the table in Section 11. Now $s \geqslant 30$ is equivalent to $t \leqslant 15$ and to $r \geqslant$ 21. If $n=8$ we get $\left(n^{2}-1\right) / 3=21$, so $\left(\log \left(1 /\left(1-n z+r z^{2}\right)\right)_{+}=n z+\left(\left(\begin{array}{c}n+1 \\ 2\end{array}\right)-r\right) z^{2}\right.$, cf. the proof of a). But from the table we get that $\left(\eta_{\bar{A}}\right)_{3}=0$ for $s=30$ and hence this is true also for $s>30$. Hence $\eta_{\bar{A}}=n z+\left(\left(\begin{array}{c}n+1 \\ 2\end{array}\right)-r\right) z^{2}=\left(\log \left(1 /\left(1-n z+r z^{2}\right)\right)_{+}\right.$ and we have proved the conjecture for all $t$ when $n=8$. If $n<8$ the proof is similar.

c) We shall prove the statement for $t \leqslant\left(\begin{array}{c}n+1 \\ 2\end{array}\right)-\left(n^{2}-1\right) / 3=\left(n^{2}+3 n+2\right) / 6$ relations. As before it suffices to find one example. We get such an example from $s$ points in $P^{n}$ if $\left(\begin{array}{c}n+2 \\ 2\end{array}\right)-s \leqslant\left(n^{2}+3 n+2\right) / 6$; i.e., if $s \geqslant\left(n^{2}+3 n+2\right) / 3$. We refer to Theorem 8.2 a). Observe that $r=s-n-1$.

d) We shall prove the statement for $t \leqslant\left(\begin{array}{c}n+1 \\ 2\end{array}\right)-\left(n^{2}-1 / 2-\frac{1}{2} \sqrt{2 n^{4}-2 n^{2}+1}\right)=$ $\left(n+1-n^{2}\right) / 2+\frac{1}{2} \sqrt{2 n^{4}-2 n^{2}+1}$ relations. As before it suffices to find one example. We get such an example from $s$ points in $P^{n}$ if $\left(\begin{array}{c}n+2 \\ 2\end{array}\right)-s \leqslant\left(n+1-n^{2}\right) / 2+$ $\sqrt{2 n^{4}-2 n^{2}+1}$; i.e., if $s \geqslant n^{2}+n+\frac{1}{2}+\frac{1}{2} \sqrt{2 n^{4}-2 n^{2}+1}$. We refer to Theorem $8.2 \mathrm{~b})$.

e) As in d) it suffices to consider $s \geqslant \frac{n^{2}}{2}+n+1-\frac{1}{10} \sqrt{5 n^{4}+20}$ points in $P^{n}$. We refer to Theorem $8.2 \mathrm{c}$ ).

f) Here we can refer to 8.2 d); i.e., 32 points in $P^{9}$.

We can also give some partial results.

Theorem 7.8. If $A$ is as in Conjecture 7.6, we have

a) $\eta_{A}(z) \equiv\left(\log \left(1 /\left(1-n z+r z^{2}\right)\right)\right)_{+} \quad\left(\bmod z^{6}\right)$ if $n \leqslant 10$.

b) $\eta_{A}(z) \equiv\left(\log \left(1 /\left(1-n z+r z^{2}\right)\right)\right)_{+} \quad\left(\bmod z^{5}\right)$ if $n \leqslant 17$.

c) $\eta_{A}(z) \equiv\left(\log \left(1 /\left(1-n z+r z^{2}\right)\right)\right)_{+} \quad\left(\bmod z^{4}\right)$ if $n \leqslant 20$.

d) $\eta_{A}(z) \equiv\left(\log \left(1 /\left(1-n z+r z^{2}\right)\right)\right)_{+}\left(\bmod z^{3}\right)$.

Proof. We use the same method as in the preceeding proof.

We end this section by reformulating the conjectures above in terms of Hopf algebras.

Conjecture 7.9. Let $B=k\left\langle X_{1}, \ldots, X_{n}\right\rangle / J$, where the variables $X_{i}$ are odd and where $J$ is generated by quadratic generic Hopf elements. Then either $B$ is a noncommutative c.i. or $\eta$ is nilpotent, where $B=U(\eta)$.

Conjecture 7.10. Let $B=k\left\langle X_{1}, \ldots, X_{n}\right\rangle / J$, where the variables $X_{i}$ are odd and where $J$ is generated by $r$ quadratic generic Hopf elements. Then $\eta(z)=(\log (1 /(1-$ $\left.\left.\left.n z+r z^{2}\right)\right)\right)_{+}$, where $B=U(\eta)$.

Using Proposition 7.3 it is easy to see that these new conjectures are equivalent to their commutative versions above. In fact, it is obvious that the old conjectures imply the new ones, since if $B$ is a quadratic Hopf algebra which is a non-commutative c.i., or its underlying Lie algebra is nilpotent, or it has the minimal possible Hilbert series, then the same is true for the generic case. 
Suppose that Conjecture 7.9 is true and consider quadratic commutative algebras $A$ defined by $t$ relations. Since $A^{!}$is a non-commutative c.i. if and only if $A$ is Koszul and $A_{3}=0$, it follows from the proof of Theorem 7.1, that the set on which $A^{!}$is a non-commutative c.i. is an intersection of countably many open sets in $\operatorname{Grass}\left(\left(\begin{array}{c}n+1 \\ 2\end{array}\right), t\right)$. Also (using Proposition 7.3), the set on which $\eta_{A}$ is nilpotent is an open set in $\operatorname{Grass}\left(\left(\begin{array}{c}n+1 \\ 2\end{array}\right), t\right)$. One of these sets must be non-empty, since if $B$ is the generic Hopf algebra defined by $\left(\begin{array}{c}n+1 \\ 2\end{array}\right)-t$ quadratic Hopf elements, then $B !$ is a member of one of the sets by Conjecture 7.9. But then also the quadratic commutative algebra defined by $t$ generic relations is a member of the corresponding set.

Suppose now that Conjecture 7.10 is true. Then for each $r$, there is a quadratic commutative algebra $A$ defined by $\left(\begin{array}{c}n+1 \\ 2\end{array}\right)-r$ relations, such that $\eta_{A}(z)$ is the correct series. But since this is the minimal possible series, it follows from Proposition 7.3 that the set of $A$ in $\operatorname{Grass}\left(\left(\begin{array}{c}n+1 \\ 2\end{array}\right),\left(\begin{array}{c}n+1 \\ 2\end{array}\right)-r\right)$ such that $\eta_{A}(z)$ has the right value is an intersection of open sets. Since this set is non-empty it contains the generic algebra A.

\section{Generic points again}

Now let us consider the coordinate ring $A$ of $s$ generic points in $P_{k}^{n}$. Let $\bar{A}=A / y$ where $y$ is a linear nonzerodivisor. If $n+1 \leqslant s<\left(\begin{array}{c}n+2 \\ 2\end{array}\right)$ then $\bar{A}(z)=1+n z+(s-$ $n-1) z^{2}$. This is the series you get if you divide a polynomial ring in $n$ variables with $\left(\begin{array}{c}n+2 \\ 2\end{array}\right)-s$ generic quadratic forms, where $s \leqslant(n+2)(n+1) / 3$ (see the proof of Theorem 7.1). Let $A^{!}=\mathrm{U}\left(\eta_{A}\right)$ and $\bar{A}^{!}=\mathrm{U}\left(\eta_{\bar{A}}\right)$. It is natural to conjecture that for any $n+1 \leqslant s<\left(\begin{array}{c}n+2 \\ 2\end{array}\right), \eta_{\bar{A}}$ behaves as the free Lie algebra on $n$ generators divided by $s-n-1$ generic quadratic Lie elements. Hence by Conjecture 7.10, we should have that $\eta_{\bar{A}}(z)=\left(\log \left(1 /\left(1-n z+(s-n-1) z^{2}\right)\right)\right)_{+}$. But since $A(z)=(1+z) \bar{A}^{!}(z)$ we should have $\eta_{A}(z)=\left(\log \left((1+z) /\left(1-n z+(s-n-1) z^{2}\right)\right)\right)_{+}$. Hence it is natural to make the following conjecture.

Conjecture 8.1. Let $A$ be the coordinate ring for $s$ generic points in $P_{k}^{n}$, where $n+1 \leqslant s<\left(\begin{array}{c}n+2 \\ 2\end{array}\right)$. Let $A^{!}=\mathrm{U}\left(\eta_{A}\right)$ and $L=\eta_{A}^{\geqslant 2}$. Then

$$
\eta_{A}(z)=(n+1) z+L(z)=\left(\log \left((1+z) /\left(1-n z+(s-n-1) z^{2}\right)\right)\right)_{+} .
$$

In particular

$\operatorname{dim}_{k} L^{3}=\max \left(\left(n^{3}-n\right) / 3-(s-n-1) n, 0\right)$.

$\operatorname{dim}_{k} L^{4}=\max \left(\left(n^{4}-n^{2}\right) / 4-(s-n-1) n^{2}+\left(\begin{array}{c}s-n \\ 2\end{array}\right), 0\right)$.

$\operatorname{dim}_{k} L^{5}=\max \left(n^{5} / 5+n^{4}+2 n^{3}+2 n^{2}+4 n / 5-s\left(n^{3}+2 n^{2}+2 n\right)+n s^{2}, 0\right)$.

Hence we conjecture that

$L^{3}=0$ if and only if $s \geqslant l_{3}$.

$L^{4}=0$ if and only if $s \geqslant l_{4}$.

$L^{5}=0$ if and only if $s \geqslant l_{5}$.

(For the definition of $l_{3}, l_{4}$ and $l_{5}$, see Definition 6.3.)

Theorem 8.2. Conjecture 8.1 is true in the following cases:

a) If $l_{3} \leqslant s<\left(\begin{array}{c}n+2 \\ 2\end{array}\right)$ and $n \leqslant 20$. 
b) If $l_{4} \leqslant s<\left(\begin{array}{c}n+2 \\ 2\end{array}\right)$ and $n \leqslant 17$.

c) If $l_{5} \leqslant s<\left(\begin{array}{c}n+2 \\ 2\end{array}\right)$ and $n \leqslant 10$.

d) If $s \leqslant 1+n+n^{2} / 4$.

e) If $n \leqslant 8$.

f) If $(n, s)=(9,32)$.

Remark. The LHS in b) is approximately $0.293 n^{2}$ and the LHS in c) is approximately $0.276 n^{2}$ for large $n$.

To prove the theorem we need a lemma.

Lemma 8.3. If $L^{i}=0$ for some set of $s$ points in $P^{n}$, then $L^{i}=0$ for $t$ generic points if $t \geqslant s$.

Proof. If $s$ squares generate $\mathcal{L}_{i}$, then also $t$ generic squares do, if $t \geqslant s$.

We need a variation of the concept of strongly free sets. We define a set of quadratic elements $\left\{y_{1}, \ldots, y_{t}\right\}$ in $k\left\langle X_{1}, \ldots, X_{n}\right\rangle$ to be $d$-strongly free if

$$
k\left\langle X_{1}, \ldots, X_{n}\right\rangle /\left(y_{1}, \ldots, y_{t}\right)(z) \equiv 1 /\left(1-n z+t z^{2}\right) \quad\left(\bmod z^{d+1}\right) .
$$

Lemma 8.4. Any subset of a d-strongly free set is d-strongly free.

Proof. This follows from the corresponding proof in [An 82].

Proof of Theorem 8.2. We have shown that $L^{3}=0$ for $s=l_{3}$ random points if $n \leqslant$ 20 (see the table in Section 11). Then Lemma 8.3 gives a). For $t=\lfloor(n+1)(n+2) / 3\rfloor$ random points we have shown that $\operatorname{dim}_{k} L^{3}=-n t+2\left(\begin{array}{c}n+2 \\ 3\end{array}\right)$ if $n \leqslant 20$ (see the table). This gives that the $t$ random squares generating $I_{X}^{\perp}$ is a 3 -strongly free set. Then of course $t$ truly generic squares constitute a 3 -strongly free set, and Lemma 8.4 shows that $s \leqslant t$ generic squares are 3 -strongly free, hence $\eta(z) \equiv$ $\left(\log \left((1+z) /\left(1-n z+(s-n-1) z^{2}\right)\right)\right)_{+} \quad\left(\bmod z^{4}\right)$ if $n \leqslant 20$. We have also shown that $L^{4}=0$ if $s=\left\lceil l_{4}\right\rceil$ if $n \leqslant 17$. Hence $L^{4}=0$ if $s \geqslant l_{4}$ and $n \leqslant 17$ by Lemma 8.3. Then $L(z) \equiv\left(\log \left((1+z) /\left(1-n z+(s-n-1) z^{2}\right)\right)\right)_{+} \quad\left(\bmod z^{5}\right)$ if $n \leqslant 17$ and b) is proved. c) is proved similarly. In [Co- Tr-Va 97] it is proved that $A_{X}$ is Koszul if $s \leqslant 1+n+n^{2} / 4$. This gives that $\left(A_{X}\right)^{!}(z)=1 /\left(1-n z+(s-n-1) z^{2}\right)$, so d) follows. For e) and f) see the table.

\section{Poincaré series of generic points}

In this section we study the Poincaré series of the coordinate ring $A_{X}$; that is, $P_{A_{X}}(x, y)=\sum \operatorname{dim}_{k} \operatorname{Tor}_{i, j}^{A_{X}}(k, k) x^{i} y^{j}$. Here, we allow any $s$, not only $s<\left(\begin{array}{c}n+2 \\ 2\end{array}\right)$. In fact, the case $s \geqslant\left(\begin{array}{c}n+2 \\ 2\end{array}\right)$ will be the easy one.

Conjecture 9.1. The Poincaré series $P_{A_{X}}(x, y)$ is rational, where $A_{X}$ is the coordinate ring of a set $X$ of $s$ generic points in $P_{k}^{n}$. 
Theorem 9.2. Conjecture 9.1 is true in the following cases.

a) If $s \geqslant\left(\begin{array}{c}n+2 \\ 2\end{array}\right)$.

b) If Conjecture 8.1 is true.

Proof. In case a) we may argue as in the proof of Theorem 5.6 to conclude that $P_{A_{X}}(x, y)=(1-x)^{n+1} /(1-x(H(x, y)-1))$, where $H(x, y)$ is the series of the homology of the Koszul complex ( $A_{X}$ is Golod).

In case b) we use the fact that, if $A=A_{X} / f$ where $f$ is some linear nonzerodivisor , then $P_{A}(x, y)$ is rational if and only if $\mathrm{U}(L)(x, y)$ is rational, where $L$ is the Lie algebra associated to $A$, [Lö 86]. If Conjecture 8.1 is true, then either $L$ is nilpotent (and hence $\mathrm{U}(L)(z)$ is rational) or $\mathrm{U}(L)(z)=\frac{1}{(1+z)^{n}\left(1-n z+(s-n-1) z^{2}\right)}$

\section{Generic forms in the exterior algebra}

If $f_{1}, \ldots, f_{t}$ are generic quadratic forms in $k\left[x_{1}, \ldots, x_{n}\right], k$ a field of characteristic 0 , there is a well-known conjecture for the Hilbert series of $A_{n, t}=$ $k\left[x_{1}, \ldots, x_{n}\right] /\left(f_{1}, \ldots, f_{t}\right)$, namely that $A_{n, t}(z)=\left(\left(1-z^{2}\right)^{t} /(1-z)^{n}\right)_{+}$. This conjecture is proved in many cases, and no counterexamples are known. When calculating examples of ideals generated by quadratic forms with the expected generic Hilbert series, experience seems to indicate that one does not have to choose all forms at random. In fact, it seems that one can almost always choose the first $n$ forms to be the squares of the variables. This made us look at the problem of Hilbert series of generic forms in the exterior algebra. It turns out that the answer to the question in the exterior algebra, which is analogous to the Hochster-Laksov result for polynomial rings, is not what we expected. Although $\operatorname{dim}_{k} \wedge^{3}(V)=10$ if $V$ is 5 -dimensional, two generic forms of degree 2 do not generate $\wedge^{3}(V)$.

Theorem 10.1. Consider the exterior algebra $E$ over a vector space with basis $\left\{e_{1}, \ldots, e_{5}\right\}$ (over any field). Let $f_{1}=c_{1} e_{1} \wedge e_{2}+c_{2} e_{1} \wedge e_{3}+c_{3} e_{1} \wedge e_{4}+c_{4} e_{1} \wedge e_{5}+c_{5} e_{2} \wedge$ $e_{3}+c_{6} e_{2} \wedge e_{4}+c_{7} e_{2} \wedge e_{5}+c_{8} e_{3} \wedge e_{4}+c_{9} e_{3} \wedge e_{5}+c_{10} e_{4} \wedge e_{5}$ and $f_{2}=c_{11} e_{1} \wedge e_{2}+c_{12} e_{1} \wedge e_{1}+$ $c_{13} e_{1} \wedge e_{4}+c_{14} e_{1} \wedge e_{5}+c_{15} e_{2} \wedge e_{3}+c_{16} e_{2} \wedge e_{4}+c_{17} e_{2} \wedge e_{5}+c_{18} e_{3} \wedge e_{4}+c_{19} e_{3} \wedge e_{5}+c_{20} e_{4} \wedge e_{5}$ be two forms in $E$. Then $\left\{e_{1} \wedge f_{1}, \ldots, e_{5} \wedge f_{1}, e_{1} \wedge f_{2}, \ldots, e_{5} \wedge f_{2}\right\}$ is linearly dependent.

Proof. It suffices to prove the theorem for generic forms (so we can suppose that the $c_{i}$ 's are algebraically independent over the prime field of $k$ ). In fact,

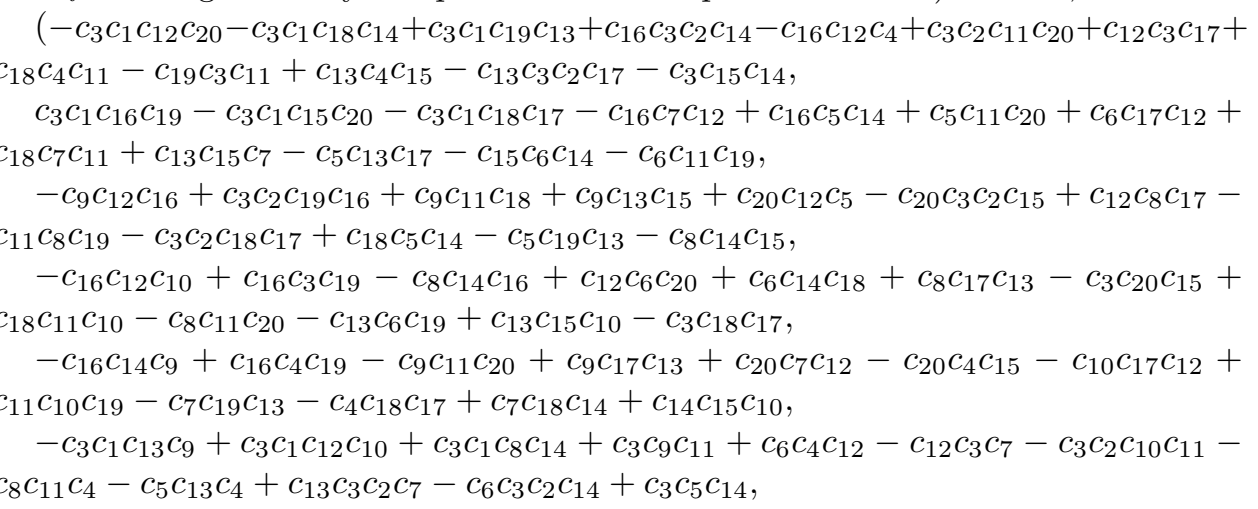




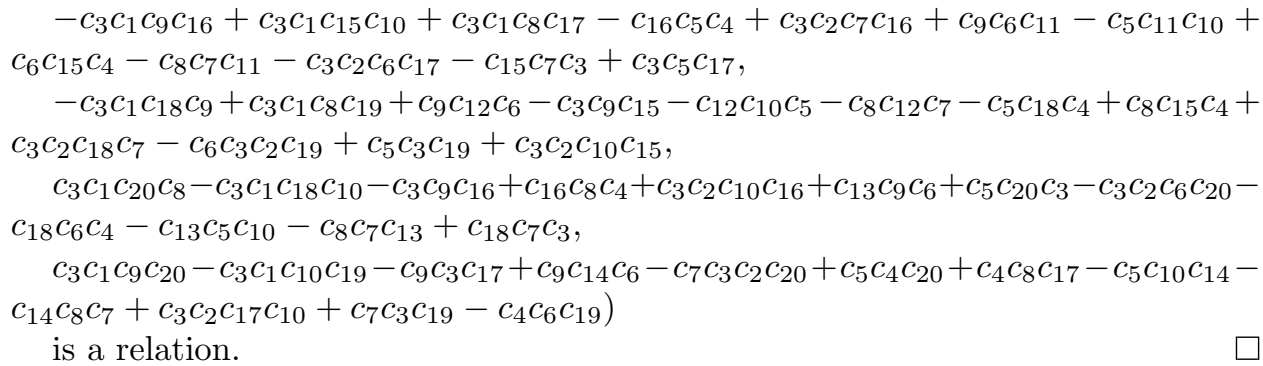

Notice that this also gives a proof of the fact that the ideal $\left(x_{1}^{2}, \ldots, x_{5}^{2}\right)$ in $S=k\left[x_{1}, \ldots, x_{5}\right]$ can not be extended in any way with two quadratic forms to an ideal $I$ with the expected generic Hilbert series $S / I(z)=1+5 z+8 z^{2}$, if $\operatorname{char}(k)=2$. It would be natural to make a conjecture about the Hilbert series of the exterior algebra modulo generic forms, similar to the one in the commutative case. The expected series for the exterior algebra in $n$ variables modulo $t$ generic quadratic forms would be $A(z)=\left((1+z)^{n}\left(1-z^{2}\right)^{t}\right)_{+}$. However, the example above shows that this is not true and there are more "probable" counterexamples given in [Gu-Sn 00], where the Hilbert series of exterior algebras modulo generic forms are discussed.

If $A$ is an exterior algebra modulo an ideal generated by quadratic forms, then $A^{!}=U\left(\eta_{A}\right)$, where $\eta_{A}$ is an ordinary Lie algebra; i.e., all generators are even. If the defining ideal of $A$ is generated by $\left(\begin{array}{l}n \\ 2\end{array}\right)-r$ quadratic forms, then $\eta_{A}$ is a free Lie algebra on $n$ generators modulo an ideal generated by $r$ quadratic elements. Recall, that if $U\left(\eta_{A}\right)=A^{!}$, then $\eta_{A}(z)=\log \left(A^{!}(z)\right)$, where $\log \left(A^{!}(z)\right)=\sum_{i \geqslant 1} e_{i} z^{i}$ if $A^{!}(z)=\prod_{i=1}^{\infty} 1 /\left(\left(1-z^{i}\right)^{e_{i}}\right.$.

One could ask when a quotient of an exterior algebra on $n$ generators modulo $t$ generic quadratic forms is Koszul. A natural guess, based on the results in Section 7 , is that this is true if and only if $t \geqslant\left(\begin{array}{l}n \\ 2\end{array}\right)-n^{2} / 4$ or $t=0$. We can prove this in one direction in the same way as in the proof of Theorem 7.1. The other direction is harder, since the example above shows that the Hochster-Laksov result cannot directly be translated to the exterior algebra case.

Theorem 10.2. Consider an exterior algebra $A$ on $n$ generators modulo $t$ linearly independent quadratic forms as a point in $\operatorname{Grass}\left(\left(\begin{array}{l}n \\ 2\end{array}\right), t\right)$. If $t \geqslant\left(\begin{array}{l}n \\ 2\end{array}\right)-n^{2} / 4$ there is a non-empty countable intersection of open sets in $\operatorname{Grass}\left(\left(\begin{array}{c}n \\ 2\end{array}\right), t\right)$ on which $A$ is Koszul. Moreover, $A$ is not Koszul if $t>0$ and $\eta_{A}$ is nilpotent, where $A^{!}=\mathrm{U}\left(\eta_{A}\right)$.

Proof. The same argument as in the proof of Theorem 7.1 shows that the set of $A$ in Grass $\left(\left(\begin{array}{l}n \\ 2\end{array}\right), t\right)$, such that $A_{3}=0$ and $A$ is Koszul, is a countable intersection of open sets. We may use the same example as before to show that this set is non-empty if $t \geqslant\left(\begin{array}{c}n \\ 2\end{array}\right)-n^{2} / 4$. Indeed, if $a=\lceil n / 2\rceil$ and $b=\lfloor n / 2\rfloor$, a simple computation shows that $\left(\begin{array}{l}a \\ 2\end{array}\right)+\left(\begin{array}{l}b \\ 2\end{array}\right)=\left\lceil\left(\begin{array}{l}n \\ 2\end{array}\right)-n^{2} / 4\right\rceil$. If $\eta_{A}$ is nilpotent and $\left(\eta_{A}\right)_{2} \neq 0$, then $1 / A^{!}(z)$ is a polynomial of degree $>n$ (see above), which is impossible if $A$ is Koszul, since $A(z)$ is a polynomial of degree $\leqslant n$.

In analogy to the case where the variables are even, it is natural to make the following conjecture. 
Conjecture 10.3. If $A$ is a generic quadratic quotient of a polynomial algebra, which is commutative in the graded sense, then $A^{!}$is either a non-commutative c.i. or $\eta_{A}$ is nilpotent.

The conjecture is false for 3 generic forms in 5 odd variables, see below. We also make the conjecture more precise in the odd case.

Conjecture 10.4. Let $A$ be an exterior algebra on $n$ variables, modulo $\left(\begin{array}{l}n \\ 2\end{array}\right)-r$ generic quadratic forms. Then $\eta_{A}(z)=\left(\log \left(1 /\left(1-n z+r z^{2}\right)\right)\right)_{+}$.

If $(n, r)=(5,7)$ or $(5,8)$, the conjecture is false, see below. We believe, however, that these are the only counterexamples.

Theorem 10.5. Conjecture 10.4 is true for $n \leqslant 8$ except for $(n, r)=(5,7),(5,8)$ when it is false. It is true also for $n=9, r \neq 21$ and in general for $r \leqslant n^{2} / 4$.

Proof. The case $r \leqslant n^{2} / 4$ follows from Theorem 10.2, since then $A^{!}(z)=1 /(1-$ $\left.n z+r z^{2}\right)$. For the other cases, it is only necessary to provide an example with the correct series in each case. We refer to the last table in Section 11. Observe that the conjecture is trivially true when $\left(\eta_{A}\right)^{3}=0$.

As in Section 7 we may reformulate the two conjectures above in terms of Hopf algebras. Consider the free associative algebra $k\left\langle X_{1}, \ldots, X_{n}\right\rangle$, where the variables have some parities $\left|X_{i}\right| \in \mathbb{Z}_{2}$. We will call an element $f=\sum c_{i j}\left[X_{i}, X_{j}\right]$ an Hopf element, where $\left[X_{i}, X_{j}\right]=X_{i} X_{j}-(-1)^{\left|X_{i} \|\right| X_{j} \mid} X_{j} X_{i}$.

Conjecture 10.6. If $B=k\left\langle X_{1}, \ldots, X_{n}\right\rangle / J$, where $J$ is generated by $r$ generic Hopf elements, then $B$ is a non-commutative c.i. or $\eta$ is nilpotent, where $B=\mathrm{U}(\eta)$.

Conjecture 10.7. If $B=k\left\langle X_{1}, \ldots, X_{n}\right\rangle / J$, where the variables are even and where $J$ is generated by $r$ generic Hopf elements, then $\eta(z)=\left(\log \left(1 /\left(1-n z+r z^{2}\right)\right)\right)_{+}$, where $B=\mathrm{U}(\eta)$.

Finally we discuss the counterexamples to Conjecture 10.4. First we consider $(n, r)=(5,7)$; i.e., 3 generic quadratic forms in 5 variables. It is shown in [Ei-Ko 94], that the forms $x_{1} x_{4}+x_{2} x_{3}, x_{1} x_{5}+x_{2} x_{4}, x_{2} x_{5}+x_{3} x_{4}$ are generic. The Lie algebra $\eta$ for this case can be determined in the same way as in [Lö-Ro 97]. The result is that $\eta(z)$ is periodic, namely $\eta(z)=(5+3 z) \sum_{i=0}^{\infty} z^{2 i+1}$, while the expected series is $5 z+3 z^{2}+5 z^{3}$. In fact, $\eta$ is the periodization of $s l(3)$ equipped with a $\mathbb{Z}_{2}$-grading. Secondly, if $(n, r)=(5,8)$; i.e., 2 generic forms in 5 variables, the expected series is $5 z+2 z^{2}$. Corollary 4.3 iii) together with Theorem 10.1 shows that $L^{3}=\eta^{3}$ is 1-dimensional, so we really have a counterexample. We also have $L^{4}=0$, so in this case the Lie algebra is nilpotent but its series is not the expected minimal one. 


\section{Tables}

We give the results of our calculations of $\eta_{A}(z)$ for the coordinate ring of $s$ random points in $P^{n}$. For the definition of $m$ and $t$, see Definition 5.3. For the definition of $l_{3}, l_{4}, l_{5}$, see Definition 6.3.

\begin{tabular}{|c|c|c|c|c|c|c|c|}
\hline$n$ & $s$ & $m$ & $t$ & $\eta_{A}(z)$ & $l_{3}$ & $l_{4}$ & $l_{5}$ \\
\hline 3 & 7 & 1 & 3 & $4 z+3 z^{2}$ & 7 & 7 & 7 \\
\hline 3 & 6 & 2 & 2 & $4 z+4 z^{2}+2 z^{3}+3 z^{4}+6 z^{5}+$. & & & \\
\hline 4 & 10 & 1 & 5 & $5 z+5 z^{2}$ & 10 & 10 & 10 \\
\hline 5 & 14 & 1 & 7 & $6 z+7 z^{2}$ & 14 & 14 & 13 \\
\hline 5 & 13 & 2 & 8 & $6 z+8 z^{2}+5 z^{3}+3 z^{4}$ & & & \\
\hline 6 & 19 & 1 & 9 & $7 z+9 z^{2}$ & 18 & 18 & 17 \\
\hline 6 & 18 & 2 & 4 & $7 z+10 z^{2}+4 z^{3}$ & & & \\
\hline 6 & 17 & 2 & 10 & $7 z+11 z^{2}+10 z^{3}+10 z^{4}$ & & & \\
\hline 7 & 24 & 1 & 12 & $8 z+12 z^{2}$ & 24 & 23 & 22 \\
\hline 7 & 23 & 2 & 7 & $8 z+13 z^{2}+7 z^{3}$ & & & \\
\hline 7 & 22 & 2 & 14 & $8 z+14 z^{2}+14 z^{3}+7 z^{4}$ & & & \\
\hline 7 & 21 & 2 & 21 & $8 z+15 z^{2}+21 z^{3}+42 z^{4}+84 z^{5}+91 z^{6}$ & & & \\
\hline 8 & 30 & 1 & 15 & $9 z+15 z^{2}$ & 30 & 28 & 27 \\
\hline 8 & 29 & 2 & 8 & $9 z+16 z^{2}+8 z^{3}$ & & & \\
\hline 8 & 28 & 2 & 16 & $9 z+17 z^{2}+16 z^{3}$ & & & \\
\hline 8 & 27 & 2 & 24 & $9 z+18 z^{2}+24 z^{3}+27 z^{4}$ & & & \\
\hline 8 & 26 & 2 & 32 & $9 z+19 z^{2}+32 z^{3}+73 z^{4}+160 z^{5}+176 z^{6}$ & & & \\
\hline 9 & 37 & 1 & 18 & $10 z+18 z^{2}$ & 37 & 34 & 33 \\
\hline 9 & 36 & 2 & 6 & $10 z+19 z^{2}+6 z^{3}$ & & & \\
\hline 9 & 34 & 2 & 24 & $10 z+21 z^{2}+24 z^{3}$ & & & \\
\hline 9 & 33 & 2 & 33 & $10 z+22 z^{2}+33 z^{3}+33 z^{4}$ & & & \\
\hline 9 & 32 & 2 & 42 & $10 z+23 z^{2}+42 z^{3}+91 z^{4}+126 z^{5}$ & & & \\
\hline 10 & 44 & 1 & 22 & $11 z+22 z^{2}$ & 44 & 41 & 39 \\
\hline 10 & 43 & 2 & 10 & $11 z+23 z^{2}+10 z^{3}$ & & & \\
\hline 10 & 41 & 2 & 30 & $11 z+25 z^{2}+30 z^{3}$ & & & \\
\hline 10 & 40 & 2 & 40 & $11 z+26 z^{2}+40 z^{3}+10 z^{4}$ & & & \\
\hline 10 & 39 & 2 & 50 & $11 z+27 z^{2}+50 z^{3}+81 z^{4}$ & & & \\
\hline 10 & 38 & 2 & 60 & $11 z+28 z^{2}+60 z^{3}+153 z^{4}+288 z^{5}+\cdots$ & & & \\
\hline 11 & 52 & 1 & 26 & $12 z+26 z^{2}$ & 52 & 48 & 46 \\
\hline 11 & 48 & 2 & 44 & $12 z+30 z^{2}+44 z^{3}$ & & & \\
\hline 11 & 47 & 2 & 55 & $12 z+31 z^{2}+55 z^{3}+25 z^{4}$ & & & \\
\hline 11 & 46 & 2 & 66 & $12 z+32 z^{2}+66 z^{3}+111 z^{4}$ & & & \\
\hline 11 & 45 & 2 & 77 & $12 z+33 z^{2}+77 z^{3}+198 z^{4}+\cdots$ & & & \\
\hline$\overline{12}$ & 61 & 1 & 30 & $13 z+30 z^{2}$ & 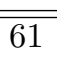 & 56 & $\overline{53}$ \\
\hline 12 & 60 & 2 & 8 & $13 z+31 z^{2}+8 z^{3}$ & & & \\
\hline 12 & 56 & 2 & 56 & $13 z+35 z^{2}+56 z^{3}$ & & & \\
\hline 12 & 55 & 2 & 68 & $13 z+36 z^{2}+68 z^{3}+3 z^{4}$ & & & \\
\hline 12 & 54 & 2 & 80 & $13 z+37 z^{3}+80 z^{3}+105 z^{4}$ & & & \\
\hline 12 & 53 & 2 & 92 & $13 z+38 z^{2}+92 z^{3}+208 z^{4}+\cdots$ & & & \\
\hline
\end{tabular}




\begin{tabular}{||r|r|r|r|l|r|r|r||}
\hline \hline$n$ & $s$ & $m$ & $t$ & $\eta_{A}(z)$ & $l_{3}$ & $l_{4}$ & $l_{5}$ \\
13 & 70 & 1 & 35 & $14 z+35 z^{2}$ & 70 & 64 & 61 \\
13 & 64 & 2 & 78 & $14 z+41 z^{2}+78 z^{3}$ & & & \\
13 & 63 & 2 & 91 & $14 z+42 z^{2}+91 z^{3}+42 z^{4}$ & & & \\
13 & 62 & 2 & 104 & $14 z+43 z^{2}+104 z^{3}+162 z^{4}$ & & & \\
13 & 61 & 2 & 117 & $14 z+44 z^{2}+117 z^{3}+283 z^{4}+\cdots$ & & & \\
\hline 14 & 80 & 1 & 40 & $15 z+40 z^{2}$ & 80 & 73 & 70 \\
14 & 73 & 2 & 98 & $15 z+47 z^{2}+98 z^{3}$ & & & \\
14 & 72 & 2 & 112 & $15 z+48 z^{2}+112 z^{3}+36 z^{4}$ & & & \\
14 & 71 & 2 & 126 & $15 z+49 z^{2}+126 z^{3}+175 z^{4}+\cdots$ & & & \\
\hline 15 & 91 & 1 & 45 & $16 z+45 z^{2}$ & 91 & 82 & 79 \\
15 & 90 & 2 & 10 & $16 z+46 z^{2}+10 z^{3}$ & & & \\
15 & 82 & 2 & 130 & $16 z+54 z^{2}+130 z^{3}$ & 102 & 92 & 88 \\
\hline 16 & 102 & 1 & 51 & $17 z+51 z^{2}$ & 114 & 103 & 98 \\
16 & 92 & 2 & 160 & $17 z+61 z^{2}+160 z^{3}$ & & & \\
\hline 17 & 114 & 1 & 57 & $18 z+57 z^{2}$ & 127 & 114 & 109 \\
17 & 103 & 2 & 187 & $18 z+68 z^{2}+187 z^{3}$ & 140 & 126 & 120 \\
\hline 18 & 127 & 1 & 63 & $19 z+63 z^{2}$ & 154 & 139 & 132 \\
18 & 126 & 2 & 12 & $19 z+64 z^{2}+12 z^{3}+\cdots$ & & & \\
\hline 19 & 140 & 1 & 70 & $20 z+70 z^{2}$ & & & \\
\hline 20 & 154 & 1 & 77 & $21 z+77 z^{2}$ &
\end{tabular}

Here are the results of our calculations of $\eta(z)$ for $r$ random quadratic relations in an ordinary Lie algebra on $n$ generators.

\begin{tabular}{||r|r|l||}
\hline \hline$n$ & $r$ & $\eta(z)$ \\
\hline 4 & 5 & $4 z+z^{2}$ \\
5 & 7 & $5 z+3 z^{2}+5 z^{3}+3 z^{4}+5 z^{5}+3 z^{6} \ldots$ \\
5 & 8 & $5 z+2 z^{2}+z^{3}$ \\
5 & 9 & $5 z+z^{2}$ \\
6 & 10 & $6 z+5 z^{2}+10 z^{3}+10 z^{4}$ \\
6 & 11 & $6 z+4 z^{2}+4 z^{3}$ \\
6 & 12 & $6 z+3 z^{2}$ \\
7 & 13 & $7 z+8 z^{2}+21 z^{3}+42 z^{4}+84 z^{5}+70 z^{6}$ \\
7 & 14 & $7 z+7 z^{2}+14 z^{3}+7 z^{4}$ \\
7 & 15 & $7 z+6 z^{2}+7 z^{3}$ \\
7 & 16 & $7 z+5 z^{2}$ \\
8 & 17 & $8 z+11 z^{2}+32 z^{3}+73 z^{4}+160 z^{5}+144 z^{6}$ \\
8 & 18 & $8 z+10 z^{2}+24 z^{3}+27 z^{4}$ \\
8 & 19 & $8 z+9 z^{2}+16 z^{3}$ \\
8 & 20 & $8 z+8 z^{2}+8 z^{3}$ \\
8 & 21 & $8 z+7 z^{2}$ \\
\hline
\end{tabular}




\begin{tabular}{||r|r|l||}
\hline \hline$n$ & $r$ & $\eta(z)$ \\
\hline 9 & 22 & $9 z+14 z^{2}+42 z^{3}+91 z^{4}+126 z^{5}$ \\
9 & 23 & $9 z+13 z^{2}+33 z^{3}$ \\
9 & 24 & $9 z+12 z^{2}+24 z^{3}$ \\
9 & 25 & $9 z+11 z^{2}+15 z^{3}$ \\
9 & 26 & $9 z+10 z^{2}+6 z^{3}$ \\
9 & 27 & $9 z+9 z^{2}$ \\
\hline \hline
\end{tabular}

\section{References}

[Ad 60]

[An 82]

[Av 74]

[Av 84]

[Ba-Ge 86]

[Be-Kr 94]

[Bo 94]

[Ca-Ro-Va 91]

[Ch-Ei 48]

[Co-Tr-Va 97]

[Ei-Ko 94]

[Ei-Po 99]

[Fr 75]
J.F. Adams, On the non-existence of elements of Hopf invariant one, Annals of Math. 72 (1960).

D. Anick, Noncommutative graded algebras and their Hilbert series, J. Algebra 78 (1982), 120-140.

L. Avramov, On the Hopf algebra of a local ring, Isv. Akad. Nauk. SSSR, Ser. mat., 38 (1974), 253-277; English translation: Math. USSR, Izv. 8 (1974).

L. Avramov, Local algebra and rational homotopy, in: Homotopie Algébrique et Algèbre Locale, Astérisque 113/114 (1984) $15-43$.

E. Ballico, A. V. Geramita, The minimal free resolution of the ideal of $s$ general points in $P^{3}$, Proc. 1984 Vancouver conf. in alg. geom., 1-10, CMS Conf. Proc. 6, AMS, Providence, 1986.

S. Beck, M. Kreuzer, How to compute the canonical module of a set of points, Algorithms in alg. geom. and appl. (Santander, 1994), Progr. Math 143, 51-78, Birkhäuser, Basel, 1996.

M. Boij, Artin level algebras, Dissertation KTH, Stockholm 1994.

M. P. Cavaliere, M. E. Rossi, G. Valla, The Green-Lazarsfeld conjecture for $n+4$ points in $P^{n}$, Rend. Sem. Mat. Univ. Pol. Torino 49 (1991), 175-195.

C. Chevalley and S. Eilenberg, Cohomology theory of Lie groups and Lie algebras, Trans. Amer. Math. Soc. 63 (1948), 85-124.

A. Conca, N. V. Trung, G. Valla, Koszul property for points in projective space, preprint 1997, acc. for publ. in Math. Scand.

D. Eisenbud, J. Koh, Nets of alternating matrices and the linear syzygy conjecture, Adv. Math. 106 (1994), 1-35.

D. Eisenbud, S. Popescu, Gale duality and free resolutions of ideals of points, Inventiones Math. 136 (1999), 419-449.

R. Fröberg, Determination of a class of Poincaré series, Math. Scand. 37 (1975), 29-39. 
[Fr-Lö 91] R. Fröberg, C. Löfwall, On Hilbert series for commutative and noncommutative graded algebras, J. Pure Appl. Algebra 76 (1991), 33-38.

[Ge-Gr-Ro 86] A. Geramita, D. Gregory, L. Roberts, Monomial ideals and points in projective space, J. Pure Appl. Algebra 40 (1986), 3362.

[Ge-Lo 89] A. V. Geramita, A. Lorenzini, The Cohen-Macaulay type of $n+3$ points in $P^{n}$, The Curves seminar at Queen's, vol. VI, no. F, Queen's Papers in Pure and Appl. Math. 83 (1989).

[Ge-Ma 84] A. V. Geramita, P. Maroscia, The ideal of forms vanishing at a finite set of points in $P^{n}$, J. Algebra 90 (1984), 528-555.

[Ge-Or 81] A. V. Geramita, F. Orecchia, On the Cohen-Macaulay type of $s$ lines in $A^{n+1}$, J. Algebra 70 (1981), 116-140.

[Gu-La-St 91] V.K.A.M. Gugenheim, L. Lambe, J. Stasheff, Perturbation theory in differential homological algebra II, Il. J. Math., 35(1991), 359-373.

[Gu-Sn 00] M.S. Guillermo, J. Snellman, Some conjectures about the Hilbert series of generic ideals in the exterior algebra, Math. $\mathrm{RA} / 0007089$.

[Gu 71] T. H. Gulliksen, A homological characterization of complete intersections, Comp. Math. 23 (1971), 251-255.

[Ha 92] S. Halperin, Universal enveloping algebras and loop space homology, J. Pure Appl. Algebra 83 (1992), 237-282.

[Hi-Si 96] A. Hirschowitz, C. Simpson, La résolution minimale de l'idéal d'un arrangement général d'un grand nombre de points dans $P^{n}$, Invent. Math. 126 (1996), 467-503.

[Ho-La 87] M. Hochster, D. Laksov, The linear syzygies of generic forms, Comm. Algebra 15 (1987), 227-239.

[Hu-Ka 91] J. Huebschmann, T. Kadeishvili, Small models for chain algebras, Math. Z. 207, No.2 (1991), 245-280.

[Jo-La 00] L. Johansson, L. Lambe, Transferring Algebra Structures up to Homology Equivalence, to appear.

[La 97] F. Lauze, Rang maximal pour $T_{P^{n}}$, Manuscripta Math. 92 (1997), 525-543.

[Lo 93] A. Lorenzini, The minimal resolution conjecture, J. Algebra 156 (1993), 5-35.

[Lö 86] C. Löfwall, On the subalgebra generated by the one-dimensional elements in the Yoneda Ext-algebra, in: Algebra, Algebraic Topology and their Interactions, Lecture Notes in Math. 1183, (Springer, Berlin 1986), 291-338.

[Lö 94] C. Löfwall, Central elements and deformations of local rings, J. Pure Appl. Algebra 91 (1994), 183-194. 
[Lö-Ro 97] C. Löfwall, J.-E. Roos, A nonnilpotent 1-2-presented graded Hopf algebra whose Hilbert series converges in the unit circle, Adv. Math. 130 (1997), 161-200.

[Ma 88] Y.I. Manin, Quantum Groups and Non-Commutative Geometry, Publ. CRM, Univ. de Montreal, 1988.

[Pr 70] X. Priddy, Koszul resolution, Trans. A.M.S. 152 (1970), 39-60.

[Wa 95] C. Walter, The minimal free resolution of the homogeneous ideal of s general points, Math. Z. 219 (1995), 231-234.

This article may be accessed via WWW at http://www.rmi.acnet.ge/hha/ or by anonymous ftp at

ftp://ftp.rmi.acnet.ge/pub/hha/volumes/2002/n2a10/v4n2a10.(dvi,ps,pdf)

R. Fröberg ralff@matematik.su.se

Department of Mathematics,

Stockholm University,

S-106 91 Stockholm,

Sweden

C. Löfwal clas@matematik.su.se

Department of Mathematics,

Stockholm University,

Sweden 